\title{
Trans-(土)-2-t-butyl-3- phenyloxaziridine: a Unique Reagent for the Oxidation of Thiolates into Sulfenates
}

Cédric Boudou, Matthieu Bergès, Charlène Sagnes, Jana

Sopková-de Oliveira Santos, Stéphane Perrio,* and Patrick

Metzner

a Laboratoire de Chimie Moléculaire et Thio-organique, ENSICAEN,

Université de Caen Basse-Normandie, CNRS, 6 Boulevard du Maréchal Juin,

14050 Caen, France

b Centre d'Etudes et de Recherche sur le Médicament de Normandie,

Université de Caen Basse-Normandie, 5 Rue Vaubénard, 14032 Caen, France

e-mail: stephane.perrio@ensicaen.fr

\section{Supporting Information}

General conditions

Typical procedure and spectral data of imines 4 and oxaziridines 1

Typical procedure and spectral data of sulfoxides 3

Synthesis of imine 6

S10

Table for the oxidation with $N$-t-butyloxaziridines 1c-e

S12

Assignment of stereochemistry for oxaziridine 1b (NMR and ORTEP

S13 diagram)

Copies of ${ }^{1} \mathrm{H}$ and ${ }^{13} \mathrm{C}$ NMR spectra 


\section{General conditions}

Dry THF, $\mathrm{CH}_{2} \mathrm{Cl}_{2}$ and toluene were displayed by a passage down an activated alumina column. $n$-Hexane and triethylamine were freshly distilled under a nitrogen atmosphere over $\mathrm{CaH}_{2}$. Chlorodiphenylphosphine was distilled at $110{ }^{\circ} \mathrm{C}$ under a reduced pressure of $6.10^{-2}$ mmHg. All other reagents and solvents were used as received from commercial sources. All reactions were performed in oven-dried glassware, under an atmosphere of dry nitrogen. The concentrations of organolithium reagents were determined by titration against diphenylacetic acid. ${ }^{1}$ Reactions were purified by chromatography column with silica gel Si $60(0.040-0.063$ $\mathrm{nm})$. Thin layer chromatography was carried out on silica gel $60 \mathrm{~F}_{254}(1.1 \mathrm{~mm})$ with spot detection under UV light or through $\mathrm{I}_{2}$ or $\mathrm{KMnO}_{4}$ oxidation. Melting points were obtained on a capillary apparatus and are uncorrected. NMR spectra were obtained in $\mathrm{CDCl}_{3}$ unless stated otherwise. All chemical shifts ( $\delta$ ) and coupling constants are quoted in parts per million (ppm) and Hertz $(\mathrm{Hz})$ respectively. The following abbreviations are used to designate the multiplicity of the signals: $\mathrm{s}=$ singlet; $\mathrm{d}=$ doublet; $\mathrm{t}=$ triplet $; \mathrm{m}=$ multiplet; $\mathrm{br}=$ broad and combinations thereof. The chemical shifts are calibrated to TMS ( $\delta \mathrm{H} 0.00)$ or residual proton and carbon resonance of the solvents $\mathrm{CDCl}_{3}(\delta \mathrm{H} 7.26$ and $\delta \mathrm{C} 77.16) .{ }^{31} \mathrm{P}$ chemical shifts are referred to external $85 \%$ phosphoric acid. IR and mass spectra were recorded on FT-IR and GC/MS/MS instruments, respectively. Only peaks of an intensity $>10 \%$ (except decisive ones) are listed for mass spectra. 1-Adamantanethiol $\mathbf{2 d}$ was prepared by reaction of 1-bromoadamantane with thiourea in the presence of $\mathrm{HBr}$, followed by $\mathrm{NaOH}$-mediated hydrolysis of the resulting isothiouronium salt. ${ }^{2}$

Kofron, W. G.; Baclawski, L. M. J. Org. Chem. 1976, 41, 1879-1880.

Dameron, A. A.; Charles, L. F.; Weiss, P. S. J. Am. Chem. Soc. 2005, 127, 8697-8704. 


\section{Synthesis of $N$-phosphinoyloxaziridine 1b}

(E)-3,3-Dimethylbutan-2-one oxime 5. ${ }^{3}$ Hydroxylamine hydrochloride (50 g, $0.72 \mathrm{~mol}$, $1.2 \mathrm{eq})$ and sodium acetate $(59.15 \mathrm{~g}, 0.72 \mathrm{~mol}, 1.2 \mathrm{eq})$ were added in water $(650 \mathrm{~mL})$. After complete dissolution, pinacolone $(83.9 \mathrm{~mL}, 0.6 \mathrm{~mol}, 1 \mathrm{eq})$ was added dropwise. The reaction mixture was allowed to stir vigorously for $18 \mathrm{~h}$ at room temperature. The resulting milky mixture was then filtered and the resulting solid was dried under reduced pressure in a desiccator containing $\mathrm{P}_{2} \mathrm{O}_{5}$ until the weight is constant. The vacuum must not be too high because of easy sublimation of the product. Oxime 5 was obtained as a white solid $(61.7 \mathrm{~g}, 0.53 \mathrm{~mol}, 89 \%)$. mp: $76{ }^{\circ} \mathrm{C}\left(\right.$ Litt. $^{3}:$ 75.5-76 $\left.{ }^{\circ} \mathrm{C}\right) .{ }^{1} \mathrm{H}$ NMR $(250 \mathrm{MHz}) \delta: 1.12(\mathrm{~s}, 9 \mathrm{H}), 1.86$ (s, 3H), no signal appearing for the $\mathrm{OH}$ proton. ${ }^{1} \mathrm{H}$ NMR (250 MHz, DMSO-d6) $\delta: 1.07$ (s, 9H), $1.72(\mathrm{~s}, 3 \mathrm{H}), 10.3$ (br s, 1H). ${ }^{13} \mathrm{C}$ NMR (63 MHz) $\delta: 10.5,27.8,37.6,164.6 . \mathrm{IR}\left(\mathrm{KBr}, \mathrm{cm}^{-1}\right)$ v: 1662, 2966, 3260, 3316. MS (EI) m/z: $116\left(\mathrm{MH}^{+}, 79\right), 100$ (53), 98 (100), 83 (31), 73 (68), 57 (79), 42 (88), 41 (66).

$N$-[(E)-1,2,2-trimethylpropylidene]-P,P-diphenylphosphinic amide $4 \mathrm{~b} .{ }^{4}$ Method A. To a solution of oxime $5(1.15 \mathrm{~g}, 10 \mathrm{mmol}, 1 \mathrm{eq})$ and triethylamine $(1.47 \mathrm{~mL}, 10.5 \mathrm{mmol}, 1.05 \mathrm{eq})$ in $n$-hexane $(30 \mathrm{~mL})$ was added dropwise at $-50{ }^{\circ} \mathrm{C}$ a solution of chlorodiphenylphosphine $(1.8 \mathrm{~mL}$, $10 \mathrm{mmol}, 1 \mathrm{eq})$ in $\mathrm{CH}_{2} \mathrm{Cl}_{2}(90 \mathrm{~mL})$. The reaction mixture was allowed to warm up slowly to $-5^{\circ} \mathrm{C}$ within $2 \mathrm{~h}$. The reaction mixture was filtered and the filtrate was concentrated. The resulting solid was purified by flash chromatography with diethyl ether as eluent to afford imine 4b as a white solid (2.03 g, $6.79 \mathrm{mmol}, 68 \%)$. Method B. To a solution of pinacolone ( $260 \mu \mathrm{L}, 2$ mmol, 2 eq) and diphenylphosphinamide (221 $\mathrm{mg}, 1 \mathrm{mmol}, 1 \mathrm{eq})$ in toluene $(10 \mathrm{~mL}), \mathrm{Ti}(\mathrm{OEt})_{4}$ (500 $\mu \mathrm{L}, 2 \mathrm{mmol}, 2 \mathrm{eq}$ ) was added dropwise while stirring. The reaction mixture was then heated

Smith, M. E.; Adkins, H. J. Am. Chem. Soc. 1938, 60, 657-663.

Huang, J.; Liu, X.; Wen, Y.; Qin, B.; Feng, X. J. Org. Chem. 2007, 72, 204-208. 
at $90{ }^{\circ} \mathrm{C}$ in an oil bath for $24 \mathrm{~h}$. The yellow mixture was cooled to room temperature and an aqueous $0.5 \mathrm{M} \mathrm{NaOH}$ solution $(10 \mathrm{~mL})$ was added slowly. The resulting white gel of titanium oxides was filtered through celite. The organic phase was separated and the aqueous phase was extracted with $\mathrm{CH}_{2} \mathrm{Cl}_{2}(3 \times 25 \mathrm{~mL})$. The combined organic extracts were washed with aqueous saturated $\mathrm{NaCl}$ solution $(10 \mathrm{~mL})$ and dried over $\mathrm{MgSO}_{4}$. Filtration and concentration under reduced pressure led to imine $4 \mathbf{b}$ as a yellow solid (266 mg, $0.89 \mathrm{mmol}, 85 \%$ ), which can be used without further purification. TLC (diethyl ether) $R_{f}=0.3 . \mathrm{mp}: 91-93{ }^{\circ} \mathrm{C}\left(\mathrm{CH}_{2} \mathrm{Cl}_{2} /\right.$ pentane). ${ }^{1} \mathrm{H}$ $\operatorname{NMR}(400 \mathrm{MHz}) \delta: 1.24(\mathrm{~s}, 9 \mathrm{H}), 2.48\left(\mathrm{~d},{ }^{4} J_{\mathrm{HP}}=2.2,3 \mathrm{H}\right), 7.39-7.45(\mathrm{~m}, 6 \mathrm{H}), 7.87-7.96(\mathrm{~m}, 4 \mathrm{H})$. ${ }^{13} \mathrm{C}$ NMR $(100 \mathrm{MHz}) \delta: 21.6\left(\mathrm{~d},{ }^{3} J_{\mathrm{CP}}=13.0\right), 27.6(\mathrm{~s}), 43.8\left(\mathrm{~d},{ }^{3} J_{\mathrm{CP}}=20.4\right), 128.2\left(\mathrm{~d},{ }^{2} J_{\mathrm{CP}}=12.4\right)$, $131.1\left(\mathrm{~d},{ }^{4} J_{\mathrm{CP}}=2.6\right), 131.4\left(\mathrm{~d},{ }^{3} J_{\mathrm{CP}}=8.7\right), 134.9\left(\mathrm{~d},{ }^{1} J_{\mathrm{CP}}=130.5\right), 197.8\left(\mathrm{~d},{ }^{2} J_{\mathrm{CP}}=12\right) .{ }^{31} \mathrm{P}$ NMR

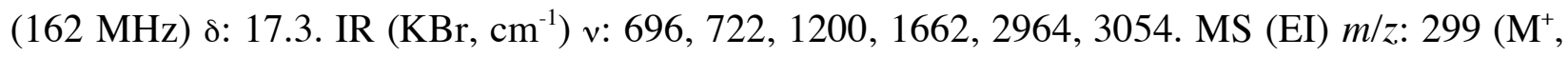
5), 284 (2), 242 (4), 202 (17), 201 (100), 77 (5). MS (CI, acetonitrile) m/z: $300\left(\mathrm{MH}^{+}, 100\right)$. Anal. Calcd for $\mathrm{C}_{18} \mathrm{H}_{22} \mathrm{NOP}$ : C, 72.22, H, 7.41, N, 4.68; Found: C, 72.37, H, 7.63, N, 4.85.

( \pm )-Trans-3-t-Butyl-3-methyl-2-(diphenylphosphinoyl)oxaziridine 1b. Potassium fluoride ( $7.1 \mathrm{~g}, 122 \mathrm{mmol}, 8 \mathrm{eq})$ was heated at $120^{\circ} \mathrm{C}$ for $2 \mathrm{~h}$ under reduced pressure (1 Torr). The Camps' complex ${ }^{5}, m$-CPBA/KF (1:2) was prepared from $\mathrm{MgSO}_{4}$ dried solution of commercial $m$-CPBA $(70 \%, 15 \mathrm{~g}, 61 \mathrm{mmol}, 4 \mathrm{eq})$ in $\mathrm{CH}_{2} \mathrm{Cl}_{2}(150 \mathrm{~mL})$ added to activated anhydrous KF previously prepared. A solution of imine $\mathbf{4 b}(4.6 \mathrm{~g}, 15.4 \mathrm{mmol}, 1 \mathrm{eq})$ in $\mathrm{CH}_{2} \mathrm{Cl}_{2}$ $(50 \mathrm{~mL})$ was then added. The white mixture was stirred at $0{ }^{\circ} \mathrm{C}$ for $3 \mathrm{~h}$ and at room temperature for a further $12 \mathrm{~h}$. The mixture was filtered and the filtrate was concentrated. The resulting solid was purified by flash chromatography with 50:50 to 0:100 pentane/diethyl ether mixtures as eluent to afford oxaziridine $\mathbf{1 b}$ as a white solid ( $3 \mathrm{~g}, 9.52 \mathrm{mmol}, 62 \%)$. TLC (diethyl ether) $R_{f}=$

\footnotetext{
$5 \quad$ Camps, F.; Coll, J.; Messeguer, A.; Pujol, F. J. Org. Chem. 1982, 47, 5402-5404.
} 
0.6. mp: $107{ }^{\circ} \mathrm{C}\left(\mathrm{CH}_{2} \mathrm{Cl}_{2} /\right.$ pentane). ${ }^{1} \mathrm{H}$ NMR (400 MHz) $\delta: 0.93$ (s, 9H), 1.95 (s, 3H), 7.46-7.93 (m, 10H). ${ }^{13} \mathrm{C}$ NMR (100 MHz) 8: $15.2\left(\mathrm{~d},{ }^{3} J_{\mathrm{CP}}=3.0\right), 25.2(\mathrm{~s}), 37.3\left(\mathrm{~d},{ }^{3} J_{\mathrm{CP}}=2.8\right), 93.0\left(\mathrm{~d},{ }^{4} J_{\mathrm{CP}}=\right.$ 7.7), $128.6\left(\mathrm{~d},{ }^{3} J_{\mathrm{CP}}=12.7\right), 130.6\left(\mathrm{~d},{ }^{1} J_{\mathrm{CP}}=120.0\right), 131.7\left(\mathrm{~d},{ }^{1} J_{\mathrm{CP}}=134.4\right), 131.8\left(\mathrm{~d},{ }^{2} J_{\mathrm{CP}}=8.9\right)$, $132.1\left(\mathrm{~d},{ }^{2} J_{\mathrm{CP}}=9.1\right), 132.2\left(\mathrm{~d},{ }^{4} J_{\mathrm{CP}}=2.8\right), 132.5\left(\mathrm{~d},{ }^{4} J_{\mathrm{CP}}=2.7\right) .{ }^{31} \mathrm{P} \mathrm{NMR}(162 \mathrm{MHz}) \delta: 28.0$. IR $\left(\mathrm{KBr}, \mathrm{cm}^{-1}\right)$ v: 1214, 1322, 1440, 2970, 3050. MS (CI, iso-butane) $m / z: 358\left[\left(\mathrm{M}+\mathrm{C}_{3} \mathrm{H}_{7}\right)^{+}, 3\right]$, $356\left[\left(\mathrm{M}+\mathrm{C}_{3} \mathrm{H}_{5}\right)^{+}, 3\right], 354\left[\left(\mathrm{M}+\mathrm{C}_{3} \mathrm{H}_{3}\right)^{+}, 4\right], 316\left(\mathrm{MH}^{+}, 100\right), 300$ (3), 244 (2), 219 (10), 201 (5), 98 (5). Anal. Calcd for $\mathrm{C}_{18} \mathrm{H}_{22} \mathrm{NO}_{2} \mathrm{P}: \mathrm{C}, 68.56, \mathrm{H}, 7.03, \mathrm{~N}, 4.44$, Found: C, 68.79, H, 7.33, N, 4.54 .

\section{Synthesis of $N$-t-butyloxaziridine 1c}

2-Methyl- $N$-methylenepropan-2-amine $4 \mathbf{c}^{6}{ }^{6} t$-Butylamine $(5.29 \mathrm{~mL}, 50 \mathrm{mmol}, 1 \mathrm{eq})$ was added to paraformaldehyde $(1.5 \mathrm{~g}, 50 \mathrm{mmol}, 1 \mathrm{eq})$ and the resulting mixture was stirred at room temperature for $5 \mathrm{~h} . \mathrm{CH}_{2} \mathrm{Cl}_{2}(20 \mathrm{~mL})$ was added and the solution was filtered. The organic layer was dried over $\mathrm{MgSO}_{4}$, filtered and concentrated under reduced pressure (be careful because of the volatility of the product) to furnish imine $\mathbf{4 c}$ as a colorless oil ( $2.84 \mathrm{~g}, 33.4 \mathrm{mmol}, 67 \%) . \mathbf{4 c}$ was used without any further purification. ${ }^{1} \mathrm{H}$ NMR $(400 \mathrm{MHz}) \delta: 1.19$ (s, 9H), 7.27 and $7.40(\mathrm{AB}$ system, $J=16.2,2 \mathrm{H}) .{ }^{13} \mathrm{C}$ NMR $(63 \mathrm{MHz}) \delta: 29.1,58.4,147.7 . \mathrm{IR}\left(\mathrm{KBr}, \mathrm{cm}^{-1}\right)$ v: 1276,1360 , 1478, 2968. MS (EI) $m / z: 85\left(\mathrm{M}^{+}, 23\right), 70$ (10).

2-(t-Butyl)oxaziridine 1c. ${ }^{7}$ The Camps' complex, $m$-CPBA/KF (1:2), was prepared as described above with commercial $m$-CPBA $(7.48 \mathrm{~g}, 33.4 \mathrm{mmol}, 1 \mathrm{eq})$ and activated anhydrous potassium fluoride $(3.87 \mathrm{~g}, 66.8 \mathrm{mmol}, 2 \mathrm{eq})$ in $\mathrm{CH}_{2} \mathrm{Cl}_{2}(300 \mathrm{~mL})$. A solution of the imine $4 \mathbf{c}$ ( $2.84 \mathrm{~g}, 33.4 \mathrm{mmol}, 1 \mathrm{eq})$ in $\mathrm{CH}_{2} \mathrm{Cl}_{2}(100 \mathrm{~mL})$ was added. The white mixture was stirred at $0{ }^{\circ} \mathrm{C}$

Vijn, R. J.; Arts, H. J.; Green, R.; Castelijns, A. M. Synthesis 1994, 573-578.

Pirkle, W. H.; Rinaldi, P. L. J. Org. Chem. 1977, 42, 3217-3219. 
for $3 \mathrm{~h}$ and at room temperature for a further $3 \mathrm{~h}$. The mixture was filtered and the filtrate was concentrated without heating because of the volatility of the product. Oxaziridine $\mathbf{4 c}$ was isolated as a colorless oil $(2.79 \mathrm{~g}, 27.6 \mathrm{mmol}, 82 \%) .{ }^{1} \mathrm{H}$ NMR (250 MHz) $\delta: 1.09(\mathrm{~s}, 9 \mathrm{H}), 3.78$ and 3.81 (AB system, $J=10,2 \mathrm{H}) .{ }^{13} \mathrm{C}$ NMR $(100 \mathrm{MHz}) \delta: 25.2,58.2,65.8 . \mathrm{IR}\left(\mathrm{NaCl}, \mathrm{cm}^{-1}\right)$ v: 1214,1270 , 1364, 1476, 2934. MS (CI, iso-butane) m/z: $102\left(\mathrm{MH}^{+}, 100\right), 86$ (4), 72 (16).

\section{Synthesis of $N$-t-butyloxaziridine 1d}

(E)-2-Methyl- $N$-(4-nitrophenylmethylene)propan-2-amine $4 d .^{8}$ In a two-necked flask equipped with a Claisen and a condenser was placed a solution of tert-butylamine (20.4 mL, 200 mmol, 4 eq) in toluene $(8 \mathrm{~mL}) \cdot p$-Nitrobenzaldehyde $(7.65 \mathrm{~g}, 50 \mathrm{mmol}, 1 \mathrm{eq})$ was then added in four aliquots to the stirred solution over a 20 min period. After stirring for $2 \mathrm{~h}$, the solution was heated until distillation of the amine excess, water and toluene is finished. Imine $\mathbf{4 d}$ was isolated as a brown solid (10.3 g, $50 \mathrm{mmol}, 100 \%)$, which can be used directly in the oxidation step. mp:

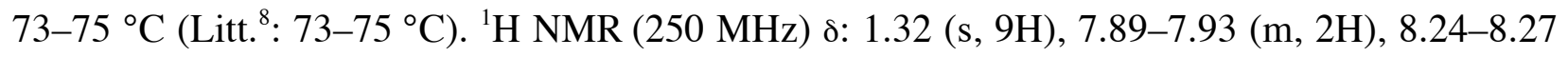

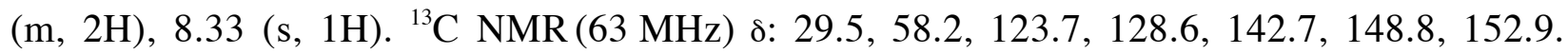
IR $\left(\mathrm{KBr}, \mathrm{cm}^{-1}\right) v: 1522,1598,1639,1684,2968,3030 . \mathrm{MS}\left(\mathrm{CI}, \mathrm{CH}_{3} \mathrm{CN}\right) \mathrm{m} / z: 207\left(\mathrm{MH}^{+}, 100\right)$, $190(8)$

(土)-Trans-2-(t-butyl)-3-(4-nitrophenyl)oxaziridine $1 \mathrm{~d} .{ }^{8}$ Following the oxidation procedure previously described, imine $4 \mathbf{d}$ (10.3 g, $50 \mathrm{mmol}, 1 \mathrm{eq})$ was treated with a suspension of potassium fluoride $(11.6 \mathrm{~g}, 200 \mathrm{mmol}, 4 \mathrm{eq})$ and commercial $m$-CPBA $(22.5 \mathrm{~g}, 100 \mathrm{mmol}, 2$ eq) in $\mathrm{CH}_{2} \mathrm{Cl}_{2}(550 \mathrm{~mL})$. Oxaziridine $\mathbf{1 d}$ was obtained after recrystallisation in pentane as a yellow solid (9.1 g, $50 \mathrm{mmol}, 82 \%)$. mp: $63-64{ }^{\circ} \mathrm{C}$, pentane (Litt. $\left.{ }^{8}: 65-66{ }^{\circ} \mathrm{C}\right) .{ }^{1} \mathrm{H}$ NMR $(250$

8 Emmons, W. D. J. Am. Chem. Soc. 1957, 79, 5739-5754. 


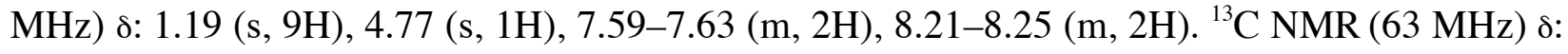
25.2, 58.9, 72.3, 123.6, 128.6, 142.7, 148.9. IR $\left(\mathrm{KBr}, \mathrm{cm}^{-1}\right) v: 1350,1521,2972,3035 . \mathrm{MS}$ (EI) $m / z: 223\left(\mathrm{MH}^{+}, 100\right), 207$ (6), 150 (12), 92 (10).

\section{Synthesis of $N$-t-butyloxaziridine 1e}

(E)-2-Methyl- $N$-(phenylmethylene)propan-2-amine 4e. ${ }^{8}$ In a two-necked flask equipped with a Claisen and a condenser was placed a solution of tert-butylamine $(20.4 \mathrm{~mL}, 200 \mathrm{mmol}, 4$ eq) in toluene $(8 \mathrm{~mL})$. Benzaldehyde $(5.1 \mathrm{~mL}, 50 \mathrm{mmol}, 1 \mathrm{eq})$ was then added in four aliquots to the stirred solution over a 20 min period. After stirring for $2 \mathrm{~h}$, the solution was heated until distillation of excess of amine, water and toluene is finished. The residue was dissolved in $\mathrm{CH}_{2} \mathrm{Cl}_{2}(4 \mathrm{~mL})$, dried over $\mathrm{MgSO}_{4}$, filtered and concentrated under reduced pressure. The crude product was then purified by Kügelrohr distillation to afford imine $4 \mathbf{e}$ as a yellow oil $(7.65 \mathrm{~g}$, $49.5 \mathrm{mmol}, 95 \%)$. bp: $90{ }^{\circ} \mathrm{C}, 10 \mathrm{mmHg}\left(\right.$ Litt. $\left.^{8}: 90-92{ }^{\circ} \mathrm{C}, 11 \mathrm{mmHg}\right) ;{ }^{1} \mathrm{H}$ NMR $(400 \mathrm{MHz}) \delta$ : $1.30(\mathrm{~s}, 9 \mathrm{H}), 7.39-7.4(\mathrm{~m}, 3 \mathrm{H}), 7.73-7.76(\mathrm{~m}, 2 \mathrm{H}), 8.27$ (s, 1H). ${ }^{13} \mathrm{C}$ NMR (63 MHz) 8: 29.7, $57.2,127.9,128.5,130.1,137.2,155.1 . \mathrm{IR}\left(\mathrm{KBr}, \mathrm{cm}^{-1}\right) v: 1650,2995,3030 . \mathrm{MS}\left(\mathrm{CI}, \mathrm{CH}_{3} \mathrm{CN}\right)$ $m / z: 162\left(\mathrm{MH}^{+}, 55\right), 146(100), 104(9), 91$ (4), $89(5), 77$ (3).

( \pm )-Trans-2-(t-butyl)-3-phenyloxaziridine $1 \mathrm{e}^{8}$ Following the previously described oxidation procedure, imine $4 \mathbf{e}(7.65 \mathrm{~g}, 47.5 \mathrm{mmol}, 1 \mathrm{eq})$ was treated with a solution of potassium fluoride (11.0 g, $190 \mathrm{mmol}, 4 \mathrm{eq})$ and commercial $m$-CPBA (21.3 g, $95 \mathrm{mmol}, 2 \mathrm{eq})$ in $\mathrm{CH}_{2} \mathrm{Cl}_{2}$ (350 mL). Oxaziridine 1e was obtained after purification by Kügelrohr distillation as a yellow oil (5.46 g, $30.8 \mathrm{mmol}, 65 \%) .{ }^{1} \mathrm{H}$ NMR (250 MHz) 8: $1.18(\mathrm{~s}, 9 \mathrm{H}), 4.68(\mathrm{~s}, 1 \mathrm{H}), 7.36-7.44(\mathrm{~m}, 5 \mathrm{H})$.

${ }^{13} \mathrm{C}$ NMR $(63 \mathrm{MHz}) \delta: 25.3,58.4,73.7,127.5,128.5,129.8,135.6 . \mathrm{IR}\left(\mathrm{KBr}, \mathrm{cm}^{-1}\right)$ v: 1362,1458 , 2972, 3035. MS (EI) m/z: 177 (M+, 16), 162 (42), 122 (11), 105 (100), 77 (31), 51 (10). 


\section{Typical procedure for the synthesis of sulfoxides via sulfenate salts.} (Benzylsulfinylmethyl)benzene 3a. ${ }^{9}$ A solution of $n$-BuLi $(760 \mu \mathrm{L}$ of a $1.47 \mathrm{~N}$ solution in hexanes, $1.12 \mathrm{mmol}, 1.05 \mathrm{eq})$ was added dropwise at $20{ }^{\circ} \mathrm{C}$ to a solution of benzyl thiol $2 \mathbf{a}$ (120 $\mu \mathrm{L}, 1 \mathrm{mmol}, 1 \mathrm{eq})$ in THF ( $5 \mathrm{~mL})$. After $15 \mathrm{~min}$, a solution of $N$-t-butyloxaziridine $1 \mathrm{e}(193 \mathrm{mg}$, $1.09 \mathrm{mmol}, 1.03 \mathrm{eq})$ in THF (2 mL) was added slowly at $20{ }^{\circ} \mathrm{C}$. After stirring the solution at 20 ${ }^{\circ} \mathrm{C}$ for $30 \mathrm{~min}$, the reaction was treated with benzyl bromide $(250 \mu \mathrm{L}, 2.1 \mathrm{mmol}, 2 \mathrm{eq})$. The resulting mixture was stirred $4 \mathrm{~h}$, then water $(10 \mathrm{~mL})$ was added and the solution was extracted with ethyl acetate $(3 \times 25 \mathrm{~mL})$. The combined organic layers were dried over $\mathrm{MgSO}_{4}$, filtered and concentrated under reduced pressure. The resulting solid was purified on silica gel with diethyl ether as eluent to afford sulfoxide 3a as a white solid (133 mg, $0.58 \mathrm{mmol}, 58 \%$ ). TLC (diethyl ether) $R_{f}=0.26 . \mathrm{mp}: 129-131^{\circ} \mathrm{C}, \mathrm{CH}_{2} \mathrm{Cl}_{2}$ (Litt. ${ }^{9}: 134^{\circ} \mathrm{C}$, benzene/diethyl ether). ${ }^{1} \mathrm{H}$ NMR (400 $\mathrm{MHz}) \delta: 3.88$ and 3.93 (AB system, $J=13.0,2 \mathrm{H}), 7.27-7.41(\mathrm{~m}, 10 \mathrm{H}) .{ }^{13} \mathrm{C} \mathrm{NMR}(63 \mathrm{MHz}) \delta$ : 57.3, 128.3, 128.9, 130.2, 130.3. IR $\left(\mathrm{KBr}, \mathrm{cm}^{-1}\right) v: 1030,2926,2956,3030 . \mathrm{MS}$ (EI) m/z: 230 $\left(\mathrm{M}^{+}, 3\right), 91(100), 65(21)$.

[(Cyclohexylsulfinyl)methyl]benzene $3 \mathbf{b}^{10}$ Following the general procedure described above with cyclohexanethiol $\mathbf{2 b}(130 \mu \mathrm{L}, 1.06 \mathrm{mmol}, 1 \mathrm{eq})$ as substrate, sulfoxide $\mathbf{3 b}$ was obtained after chromatography purification (diethyl ether) as a white solid (99 mg, $0.45 \mathrm{mmol}$, 42\%). TLC (diethyl ether) $R_{f}=0.3 . \mathrm{mp}: 82-84{ }^{\circ} \mathrm{C}$ (Litt. ${ }^{10}: 84-85^{\circ} \mathrm{C}$, benzene/petroleum ether). ${ }^{1} \mathrm{H} \operatorname{NMR}(250 \mathrm{MHz}) \delta: 1.20-1.91(\mathrm{~m}, 10 \mathrm{H}), 2.45(\mathrm{tt}, J=3.5$ and $11.7,1 \mathrm{H}), 3.90$ and $3.98(\mathrm{AB}$ system, $J=13.0,2 \mathrm{H}), 7.28-7.39(\mathrm{~m}, 5 \mathrm{H}) .{ }^{13} \mathrm{C} \mathrm{NMR}(63 \mathrm{MHz})$ 8: 24.3, 25.5, 25.8, 25.9, 27.3,

\footnotetext{
$9 \quad$ Rajanikanth, B.; Ravindranath, B. Indian J. Chem. Sect. B 1984, 23, 877-878.
}

$10 \quad$ Weibull, B. Arkiv. Kemi 1951, 3, 171-193. 
55.2, 57.3, 128.5, 129.3, 130.3, 131.0. IR $\left(\mathrm{KBr}, \mathrm{cm}^{-1}\right) v: 1024,2914,3035$. MS (EI) $m / z: 223$ $\left(\mathrm{MH}^{+}, 36\right), 140$ (24), 91 (100), 65 (17), 55 (16).

[(t-Butylsulfinyl)methyl]benzene 3c. ${ }^{9}$ Following the general procedure described above with $t$-butanethiol $\mathbf{2 c}(120 \mu \mathrm{L}, 1.06 \mathrm{mmol}, 1 \mathrm{eq})$ as substrate, sulfoxide $\mathbf{3 c}$ was obtained after chromatography purification (pentane/diethyl ether, 20:80) as a white solid (90 mg, $0.43 \mathrm{mmol}$, 43\%). TLC (diethyl ether) $R_{f}=0.25 . \mathrm{mp}: 74-75{ }^{\circ} \mathrm{C}$, pentane (Litt. ${ }^{9}: 71{ }^{\circ} \mathrm{C}$, benzene/diethyl ether). ${ }^{1} \mathrm{H}$ NMR (250 MHz) $\delta: 1.33$ (s, 9H), 3.63 and 3.83 (AB system, $\left.J=12.8,2 \mathrm{H}\right), 7.32-7.38$ (m, 5H). ${ }^{13} \mathrm{C}$ NMR $(100.6 \mathrm{MHz}) \delta: 23.2,53.1,53.8,128.1,129.1,130.1,132.1 . \mathrm{IR}\left(\mathrm{KBr}, \mathrm{cm}^{-1}\right) v$ : 770, 1034, 2978, 3032. MS (CI, iso-butane) $\mathrm{m} / z: 235\left[\left(\mathrm{M}+\mathrm{C}_{3} \mathrm{H}_{3}\right)^{+}, 3\right], 197\left(\mathrm{MH}^{+}, 100\right), 181$ (20), $171(9), 154$ (46), 113 (6), 107 (5), 93 (5), 91 (6).

[(1-Adamantylsulfinyl)methyl]benzene 3d. Following the general procedure described above but at $0{ }^{\circ} \mathrm{C}$ with 1 -adamantanethiol $2 \mathbf{d}(160 \mathrm{mg}, 0.95 \mathrm{mmol}, 1 \mathrm{eq})$ as substrate, sulfoxide 3d was obtained after chromatography purification (pentane/diethyl ether, 50:50) as a white solid (136 mg, $0.5 \mathrm{mmol}, 52 \%)$. TLC (diethyl ether) $R_{f}=0.36 . \mathrm{mp}: 148-150{ }^{\circ} \mathrm{C} .{ }^{1} \mathrm{H}$ NMR $(400 \mathrm{MHz})$

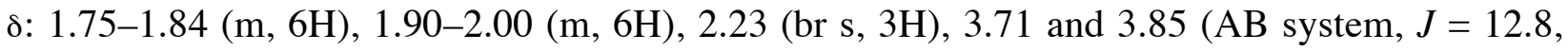

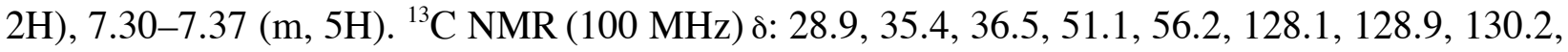
132.2. IR (KBr, $\left.\mathrm{cm}^{-1}\right)$ v: 1020, 2900, 3030. MS (CI, iso-butane) $m / z: 313\left[\left(\mathrm{M}+\mathrm{C}_{3} \mathrm{H}_{3}\right)^{+}, 4\right], 275$ ( $\left.\mathrm{MH}^{+}, 100\right), 259$ (24), 199 (4), 135 (23), 107 (11), 91 (5), 79 (13). HRMS (CI, iso-butane) Calcd for $\mathrm{C}_{17} \mathrm{H}_{23} \mathrm{OS}$ : 275.1470, Found: 275.1458.

[(Butylsulfinyl)methyl]benzene 3e. ${ }^{9}$ Following the general procedure at $20{ }^{\circ} \mathrm{C}$ with n-butanethiol $2 \mathbf{e}(110 \mu \mathrm{L}, 1 \mathrm{mmol}, 1 \mathrm{eq})$ as substrate, sulfoxide $3 \mathbf{e}$ was obtained after chromatography purification (pentane/AcOEt, 1:1) as a white solid (60 $\mathrm{mg}, 0.31 \mathrm{mmol}, 31 \%)$. 
TLC (pentane/AcOEt, 1:1) $R_{f}=0.27 . \mathrm{mp}: 61-63{ }^{\circ} \mathrm{C}$ (Litt. ${ }^{9}: 62{ }^{\circ} \mathrm{C}$, benzene/diethyl ether). ${ }^{1} \mathrm{H}$ NMR (400 MHz) 8: $0.92(\mathrm{t}, J=7.5,3 \mathrm{H}), 1.40-1.47(\mathrm{~m}, 2 \mathrm{H}), 1.69-1.75(\mathrm{~m}, 2 \mathrm{H}), 2.56(\mathrm{t}, J=7.5$, 2H), 3.9 and 4.1 (AB system, $J=12.5,2 \mathrm{H}), 7.26-7.40(\mathrm{~m}, 5 \mathrm{H}) .{ }^{13} \mathrm{C}$ NMR $(63 \mathrm{MHz})$ 8: 13.7, 22.0, 24.5, 50.7, 58.3, 128.3, 129.0, 130.0, 130.1. IR $\left(\mathrm{KBr}, \mathrm{cm}^{-1}\right) v: 1029,2936,2980,3035 . \mathrm{MS}$ (EI) $m / z: 196\left(\mathrm{M}^{+}, 2\right), 91(100), 65(14)$.

1-Benzylsulfinyl-4-methylbenzene 3f. ${ }^{11}$ Following the general procedure at $20{ }^{\circ} \mathrm{C}$ with $p$ toluenethiol $2 \mathbf{f}$ (128 $\mathrm{mg}, 1 \mathrm{mmol}, 1 \mathrm{eq})$ as substrate, sulfoxide $\mathbf{3 f}$ was obtained after chromatography purification (pure $\mathrm{CH}_{2} \mathrm{Cl}_{2}$ followed by $\mathrm{CH}_{2} \mathrm{Cl}_{2} / \mathrm{AcOEt}, 9: 1$ ) as a white solid (143 mg, $61 \mathrm{mmol}, 60 \%)$. TLC $\left(\mathrm{CH}_{2} \mathrm{Cl}_{2} / \mathrm{AcOEt}, 80: 20\right) R f=0.43 . \mathrm{mp} 139-140{ }^{\circ} \mathrm{C}$ (Litt.: ${ }^{11}$ 140-141 $\left.{ }^{\circ} \mathrm{C}\right) .{ }^{1} \mathrm{H}$ NMR $\left(250 \mathrm{MHz}, \mathrm{CDCl}_{3}\right): \delta 2.39(\mathrm{~s}, 3 \mathrm{H}), 3.97$ and $4.09(\mathrm{AB}, J=12.5,2 \mathrm{H})$, 6.97-7.01 (m, 2H), 7.20-7.89 (m, 7H). ${ }^{13} \mathrm{C}$ NMR $\left(62.9 \mathrm{MHz}, \mathrm{CDCl}_{3}\right): \delta 21.4,63.7,124.5,128.2$, 128.4, 129.4, 129.6, 130.4, 139.7, 141.6. IR $\left(\mathrm{KBr}, \mathrm{cm}^{-1}\right): v$ 3058, 3032, 2960, 2910, 1033. MS (CI, isobutane) $m / z 271\left[\left(\mathrm{M}+\mathrm{C}_{3} \mathrm{H}_{5}\right)^{+}, 2\right], 269\left[\left(\mathrm{M}+\mathrm{C}_{3} \mathrm{H}_{3}\right)^{+}, 2\right], 231\left(\mathrm{MH}^{+}, 100\right), 215$ (6).

\section{$N$-[(E)-2,2-Dimethyl-1-(2-phenylethyl)propylidene]-P,P-diphenylphosphinic amide 6.}

To a solution of $N$-phosphinoylimine $4 \mathbf{b}(299 \mathrm{mg}, 1 \mathrm{mmol}, 1 \mathrm{eq})$ in THF $(5 \mathrm{~mL})$ cooled to $-78{ }^{\circ} \mathrm{C}$ was added dropwise $n$-BuLi ( $690 \mu \mathrm{L}$ of a $1.52 \mathrm{~N}$ solution in hexanes, $1.05 \mathrm{mmol}, 1.05 \mathrm{eq}$ ). After stirring for $25 \mathrm{~min}$, the solution was allowed to warm to $0{ }^{\circ} \mathrm{C}$ over a period of $15 \mathrm{~min}$ and then recooled to $-78{ }^{\circ} \mathrm{C}$. The reaction was treated with the benzyl bromide ( $\left.230 \mu \mathrm{L}, 2 \mathrm{mmol}, 2 \mathrm{eq}\right)$. After stirring for further $15 \mathrm{~min}$, the resulting mixture was allowed to warm to room temperature and stirred for $16 \mathrm{~h}$. Water $(5 \mathrm{~mL})$ was added and the solution was extracted with diethyl ether (3 $\times 25 \mathrm{~mL}$ ). The combined organic layers were successively washed with aqueous saturated $\mathrm{NaCl}$

$11 \quad$ Kise, M.; Oae, S. Bull. Chem. Soc. Jpn. 1970, 43, 1426-1430. 
solution $(10 \mathrm{~mL})$, dried over $\mathrm{MgSO}_{4}$, filtered and concentrated under reduced pressure. The resulting solid was then purified on silica gel with pentane/diethyl ether (30:70) as eluent to afford imine 6 as a white solid (157 mg, $0.40 \mathrm{mmol}, 40 \%)$. TLC (pentane/diethyl ether, 50:50) $R_{f}$ $=0.18 . \mathrm{mp}: 97-99{ }^{\circ} \mathrm{C} .{ }^{1} \mathrm{H}$ NMR $(250 \mathrm{MHz}) \delta: 1.28(\mathrm{~s}, 9 \mathrm{H}), 2.79-2.86(\mathrm{~m}, 2 \mathrm{H}), 3.07-3.11(\mathrm{~m}$, 2H), 7.14-7.45 (m, 11H), 7.95-8.03 (m, 4H). ${ }^{13} \mathrm{C}$ NMR (63 MHz) 8: 27.7, 35.2, $37.6\left(\mathrm{~d},{ }^{3} \mathrm{~J}_{\mathrm{CP}}=\right.$ $11.9), 44.3\left(\mathrm{~d},{ }^{3} \mathrm{~J}_{\mathrm{CP}}=20.7\right), 126.1,128.4\left(\mathrm{~d},{ }^{3} \mathrm{~J}_{\mathrm{CP}}=16.9\right), 128.4,128.5,131.2\left(\mathrm{~d},{ }^{4} \mathrm{~J}_{\mathrm{CP}}=2.5\right), 131.5$ $\left(\mathrm{d},{ }^{2} \mathrm{~J}_{\mathrm{CP}}=8.8\right), 135.3\left(\mathrm{~d},{ }^{1} \mathrm{~J}_{\mathrm{CP}}=131.5\right), 141.2,198.9\left(\mathrm{~d},{ }^{2} \mathrm{~J}_{\mathrm{CP}}=12\right) .{ }^{31} \mathrm{P} \mathrm{NMR}(162 \mathrm{MHz}) \delta: 17.1 . \mathrm{IR}$ $\left(\mathrm{KBr}, \mathrm{cm}^{-1}\right)$ v: 1194, 1654, 2968, 3024. MS (EI) m/z: 389 (M+1 100), 201 (49), 91 (13), 77 (4), 65 (4). HRMS (EI) Calcd for $\mathrm{C}_{25} \mathrm{H}_{28} \mathrm{NOP}$ : 389.1908. Found: 389.1899. 
TABLE 1. Oxidation with $N$-(t-butyl)oxaziridines 1c-e

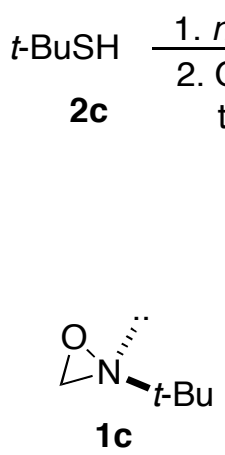

$1 c$

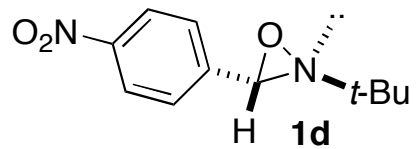

$\stackrel{\text { 3. } \mathrm{BnBr}}{\longrightarrow} t-\mathrm{BuS}(\mathrm{O}) \mathrm{Bn}$

3c

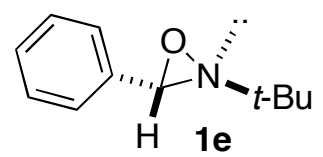

\begin{tabular}{|c|c|c|c|c|}
\hline & & \multicolumn{3}{|c|}{$\begin{array}{c}\text { Isolated yield in sulfoxide 3c with } \\
\text { oxaziridines 1c-d }\end{array}$} \\
\hline entry & $\mathrm{T}\left({ }^{\circ} \mathrm{C}\right)$ & $\mathbf{1 c}$ & $\mathbf{1 d}$ & $\mathbf{1 e}$ \\
\hline 1 & -78 & 0 & 0 & 0 \\
\hline 2 & -40 & 38 & 25 & 0 \\
\hline 3 & -40 & $46^{\mathrm{b}}$ & & \\
\hline 4 & -30 & & 43 & \\
\hline 5 & -20 & & 42 & trace \\
\hline 6 & -10 & & & 29 \\
\hline 7 & 0 & 33 & & 41 \\
\hline 8 & 20 & & & 60 \\
\hline
\end{tabular}

${ }^{\mathrm{a}} \mathrm{BnBr}(2 \mathrm{eq}) .{ }^{\mathrm{b}}$ Slow addition over $3 \mathrm{~h}$ with a syringe pump. 


\section{Assignment of stereochemistry for oxaziridine 1b}

\section{1) NMR analysis}

The ring methyl group appears at $\delta 1.95 \mathrm{ppm}$ as a singlet typical of a cis relationship with the diphenylphosphinoyl substituent. A trans location should give a doublet with a ${ }^{4} \mathbf{J}_{\mathrm{PH}}$ coupling constant of $1.7 \mathrm{~Hz}$ at $\delta$ around $1.6 \mathrm{ppm}$. See for example the data recorded for the $N$ phosphinoyloxaziridine derived from butan-2-one formed as a mixture of cis and trans stereoisomers (ratio 1:3): Cook, S. D.; Hamor, T. A.; Jennings, W. B.; Tebbutt, A. A.; Watson, S. P.; Boyd, D. R. J. Chem. Soc., Perkin Trans. 2 1991, 1281-1285.

\section{2) ORTEP diagram}

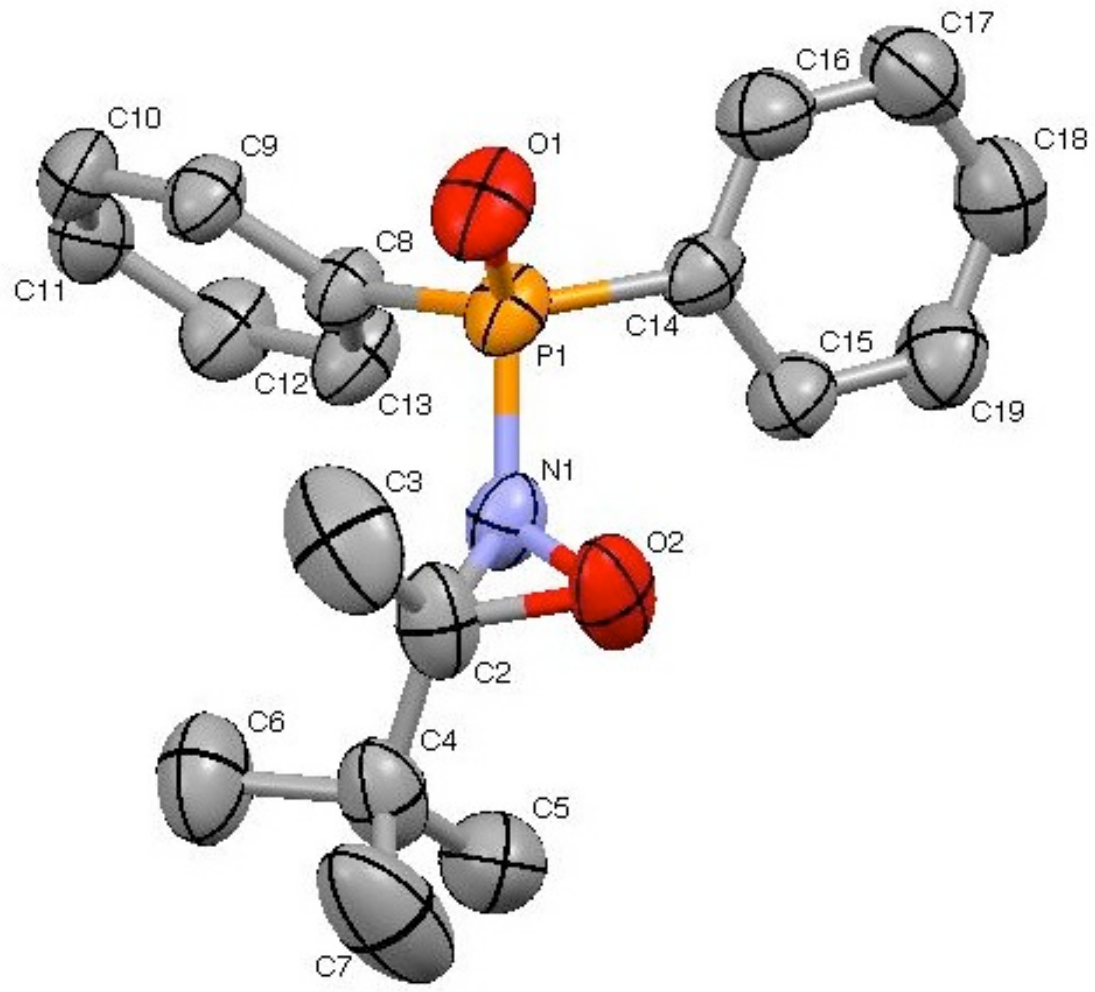


Crystal structure determination of $\mathbf{1 b}$. Single crystals of oxaziridine $1 \mathrm{~b}$ suitable for Xray crystallographic analysis were obtained by slow diffusion of pentane in $\mathrm{CH}_{2} \mathrm{Cl}_{2}$ solution. $\mathrm{X}$ ray diffraction experiments for monocrystal of $\mathbf{1 b}$ were performed at $293.2 \mathrm{~K}$ with graphite-monochromatized Mo $\mathrm{K}_{\alpha}$ radiation on an Enraf-Nonius CAD-4 diffractometer. Formula $\mathrm{C}_{18} \mathrm{H}_{22} \mathrm{NO}_{2} \mathrm{P}$, formula weight 315.34 , crystal system orthorhombic, space group Pna21, a $=12.6281(7) \AA, b=15.8244(1) \AA, c=8.7576(7) \AA, \alpha=90^{\circ}, \beta=90^{\circ}, \gamma=90^{\circ}, V=1750.0(2) \AA^{3}$, $\mathrm{Z}=4$, density calcd $=1.197 \mathrm{~g} / \mathrm{cm}^{3}, \mu=0.163 \mathrm{~mm}^{-1}, \mathrm{R}=0.0470, \mathrm{wR}=0.1311$. Selected bond lengths (Å) and angles (deg): P1-O1 1.475(2), P1-N1 1.703(9), P1-C14 1.793(2), P1-C8 1.802(2), N1-C2 1.442(3), N1-O2 1.509(3), C2-O2 1.426(3), C2-C3 1.498(4), C2-C4 1.529(4), O1-P1-N1 121.92(1), O1-P1-C8 113.18(1), N1-P1-C8 97.54(1), O1-P1-C14 112.41(1), N1-P1-C14 101.33(9), C8-P1-C14 108.78(1), C2-N1-P1 127.23(2), N1-C2-C3 119.8(3), N1-C2-C4 114.1(2), C3-C2-C4 117.9(3). Data reduction: JANA98. Program(s) used to solve structure: SHELXS-97. Program(s) used to refine structure: SHELXS-97. Software used to prepare material for publication: SHELXS-97. Crystallographic data for compound $\mathbf{1 b}$ have been deposited at the Cambridge Crystallographic Data Centre, CCDC No 642260. Copies of this information may be obtained free of charge from The Director, CCDC, 12 Union Road, Cambridge, CB2 1EZ, UK (+44-1223-336408; E-mail: deposit@ccdc.cam.ac.uk or http://www.ccdc.cam.ac.uk). 


\section{${ }^{1} \mathrm{H}$ NMR $\left(\mathrm{CDCl}_{3}, 250 \mathrm{MHz}\right)$ of 5}
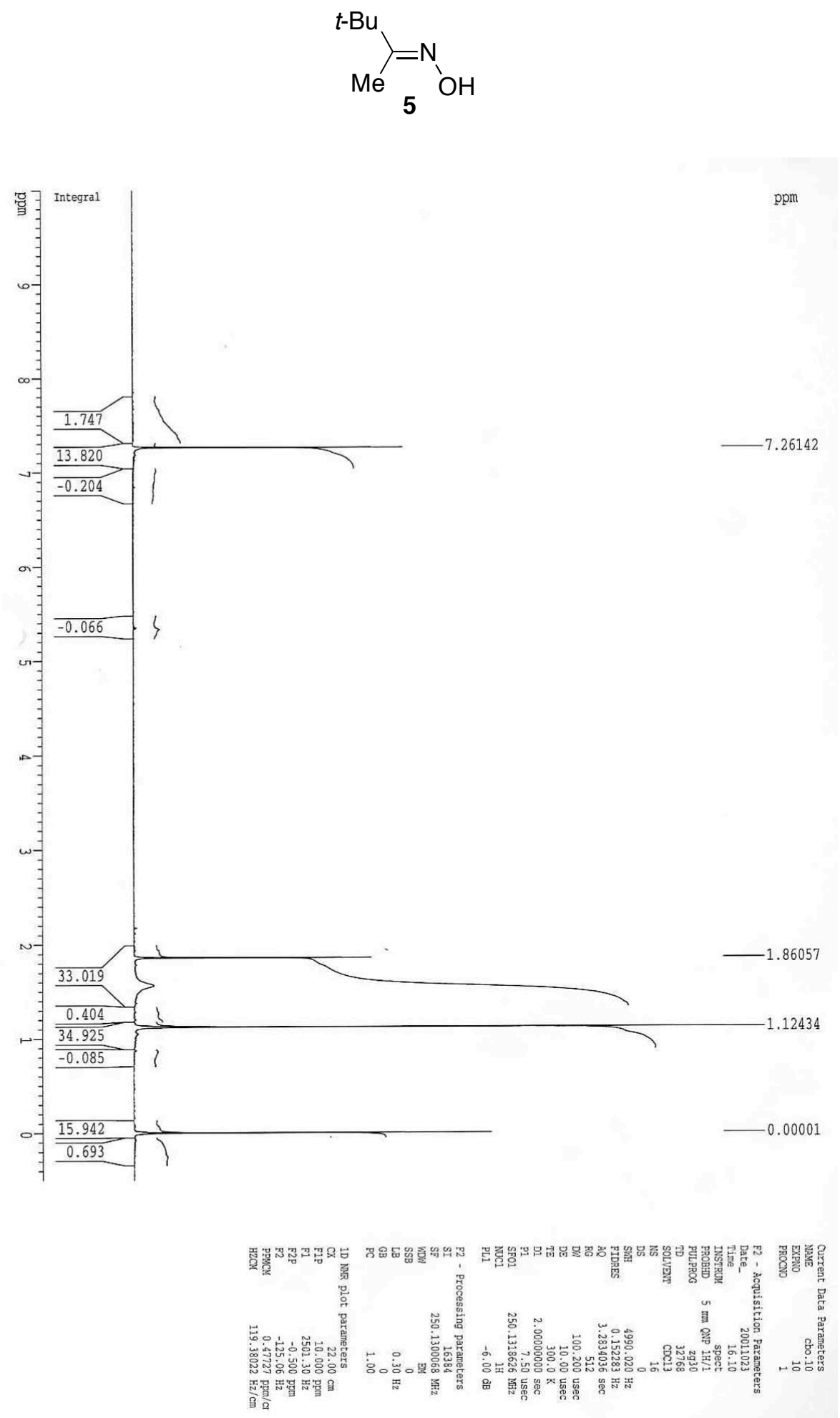
${ }^{13} \mathrm{C}$ NMR $\left(\mathrm{CDCl}_{3}, 100 \mathrm{MHz}\right)$ of 5

${ }_{\mathrm{Me}}^{t-\mathrm{Bu}}=\underset{5}{\mathrm{O}}$

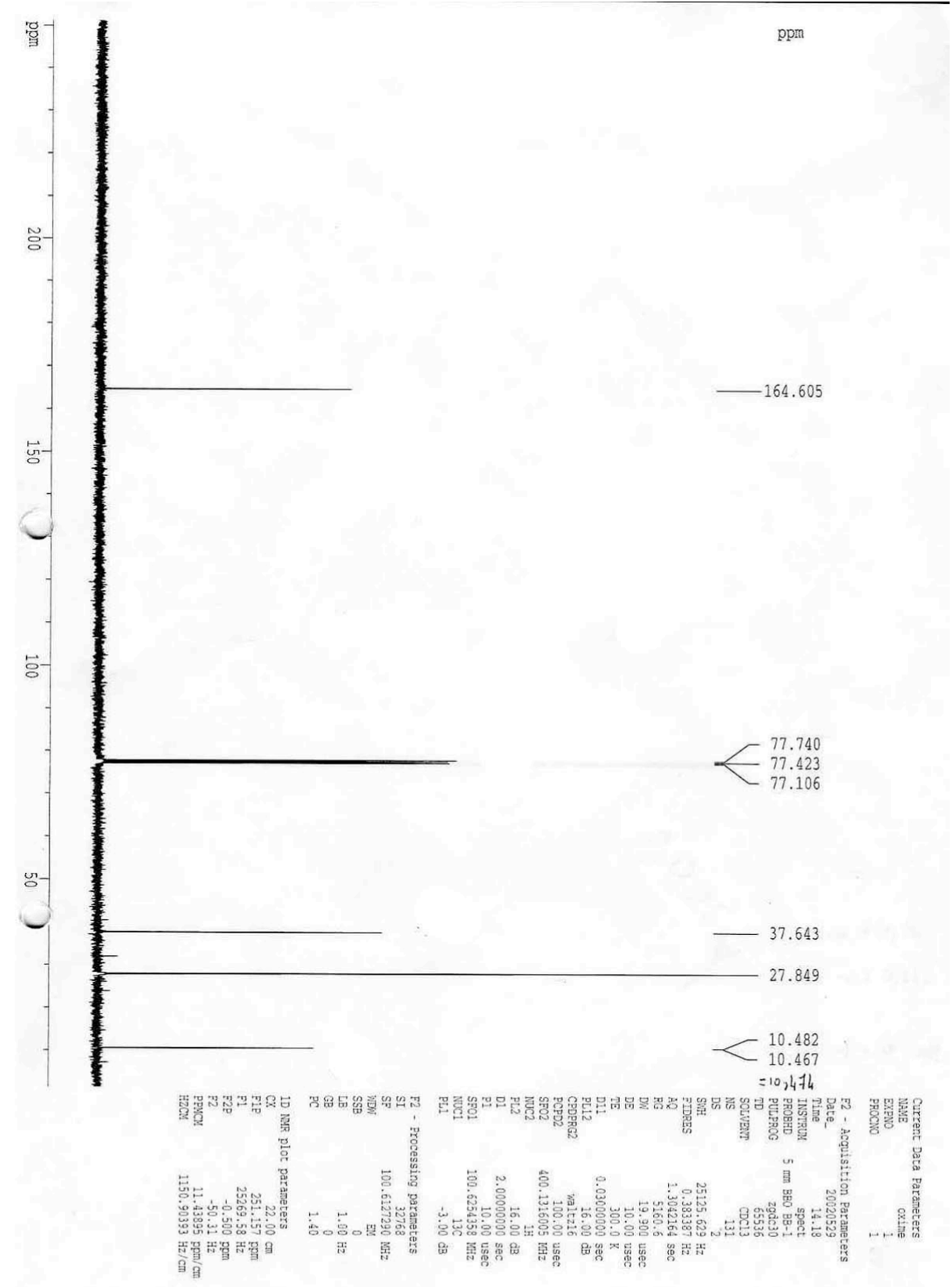




\section{${ }^{1} \mathrm{H}$ NMR $\left(\mathrm{CDCl}_{3}, 250 \mathrm{MHz}\right)$ of $4 \mathrm{~b}$}

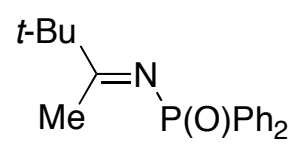

4b

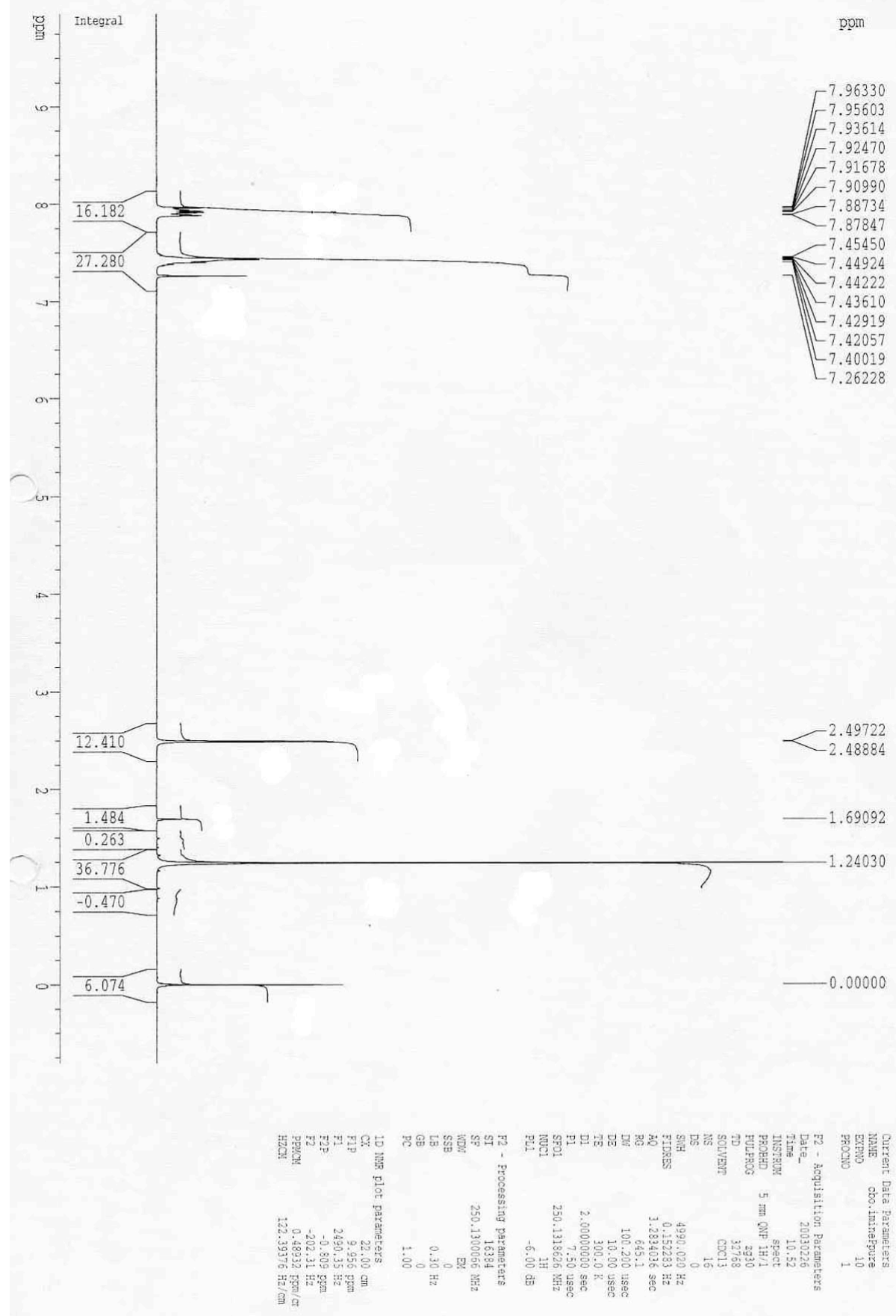


${ }^{13} \mathrm{C} \mathrm{NMR}\left(\mathrm{CDCl}_{3}, 100 \mathrm{MHz}\right)$ of $4 \mathrm{~b}$<smiles>C/C(=N\[PH](=O)c1ccccc1)C(C)(C)C</smiles>

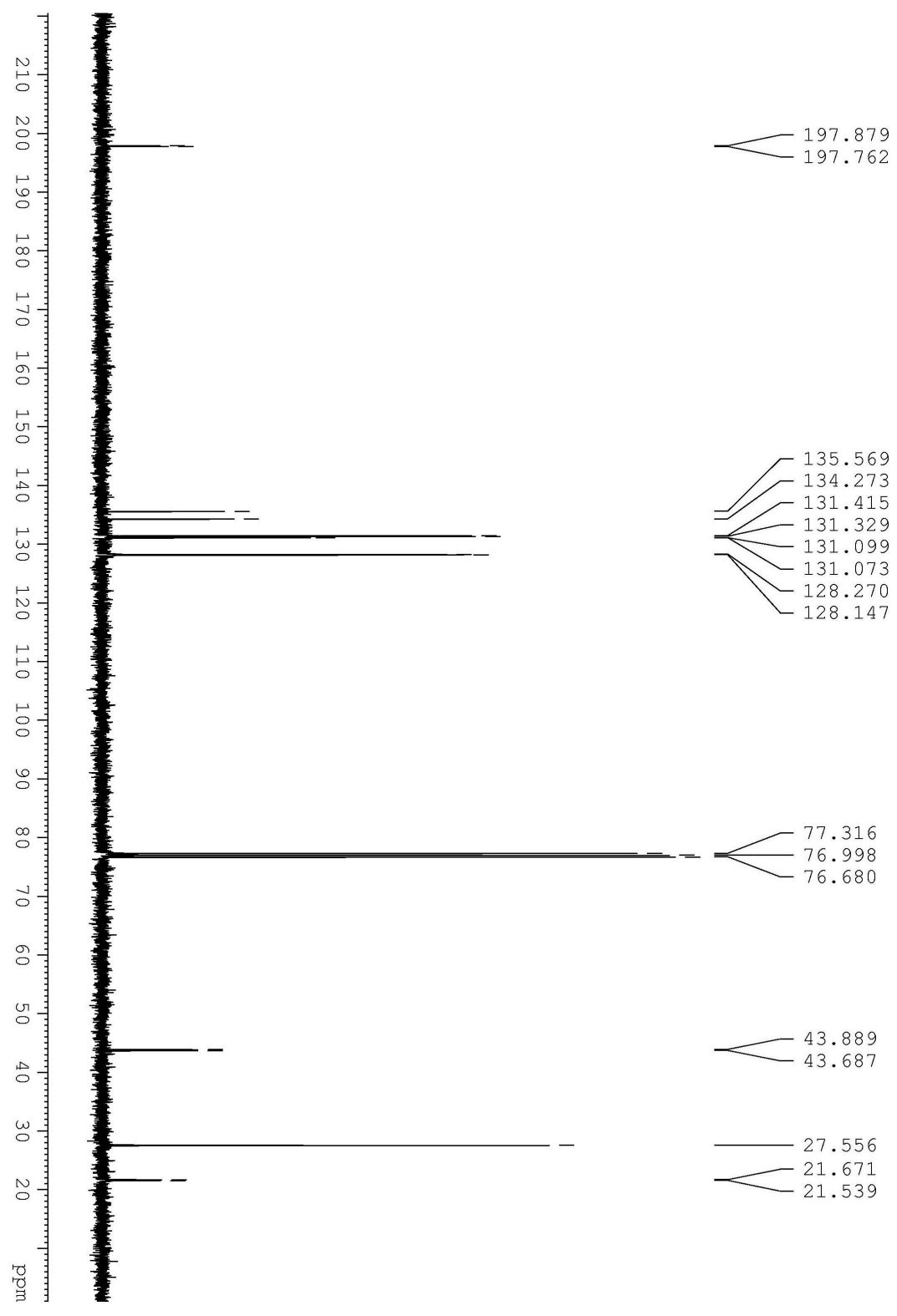


${ }^{31} \mathrm{P}$ NMR $\left(\mathrm{CDCl}_{3}, 161.9 \mathrm{MHz}\right)$ of $4 \mathrm{~b}$
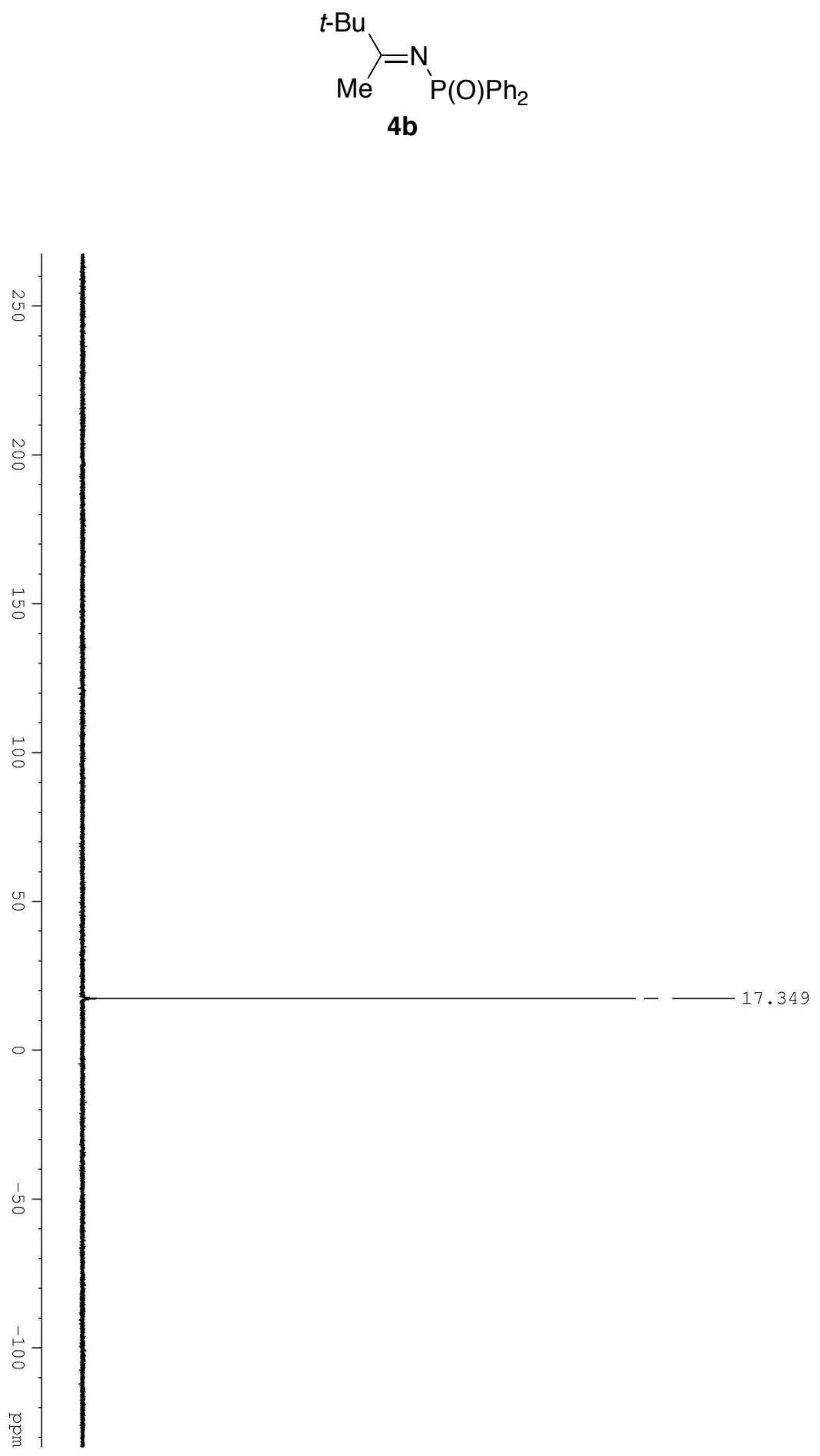


\section{${ }^{1} \mathrm{H}$ NMR $\left(\mathrm{CDCl}_{3}, 400 \mathrm{MHz}\right)$ of $1 \mathrm{~b}$}
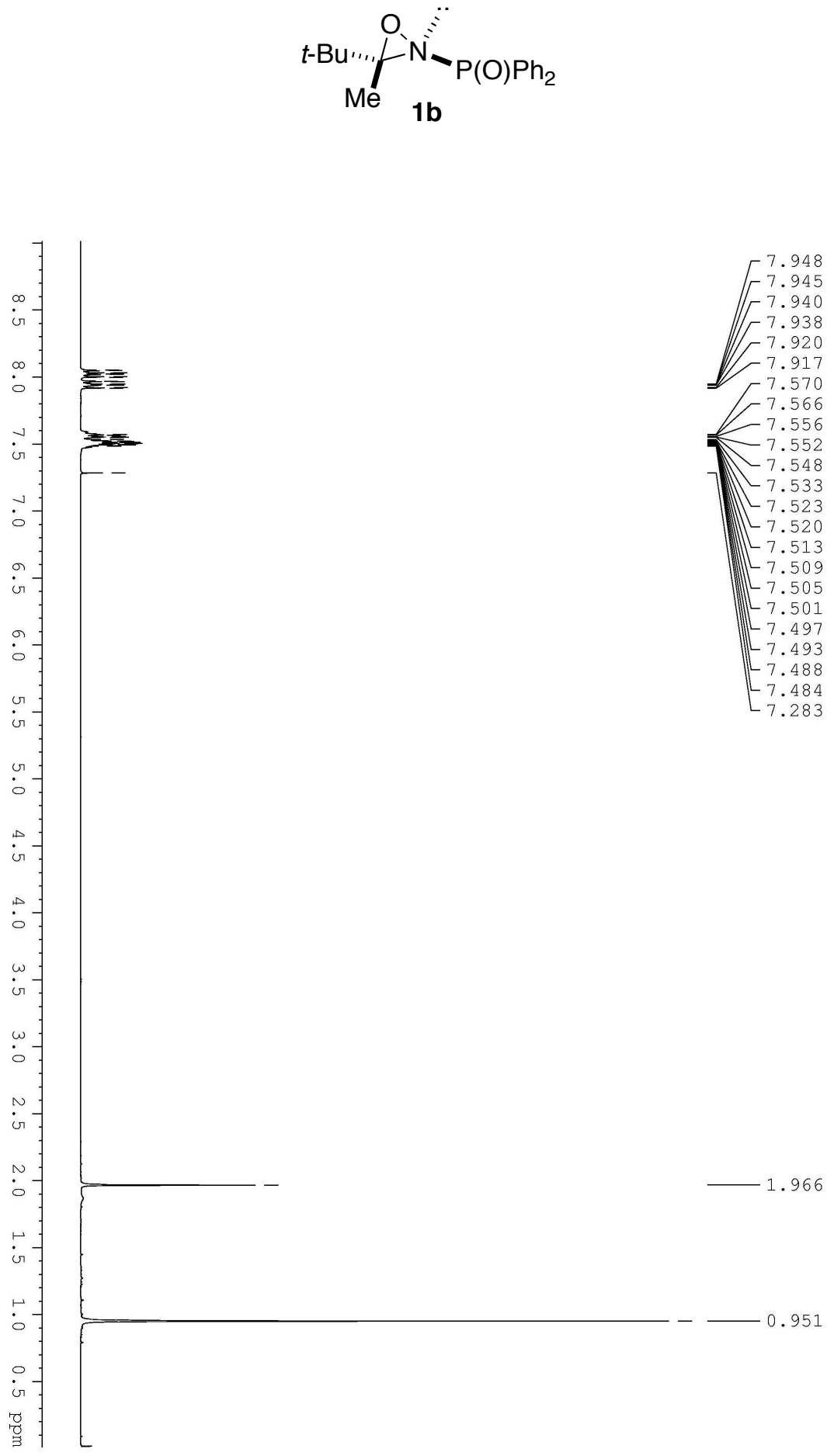


\section{${ }^{13} \mathrm{C}$ NMR $\left(\mathrm{CDCl}_{3}, 63 \mathrm{MHz}\right)$ of $1 \mathrm{~b}$}
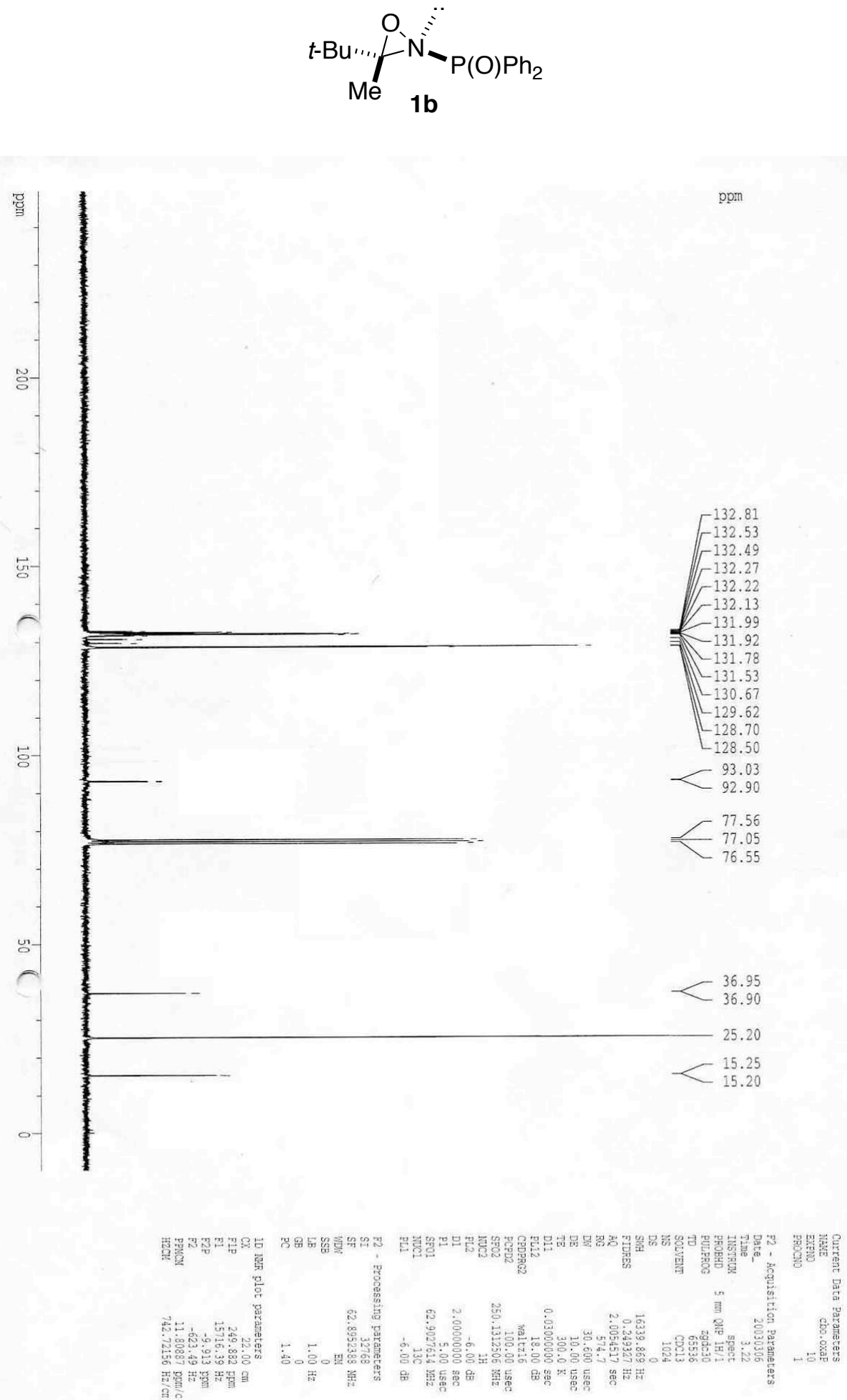


\section{Expansion of ${ }^{13} \mathrm{C}$ NMR of $1 \mathrm{~b}$ 128-133 ppm Region}
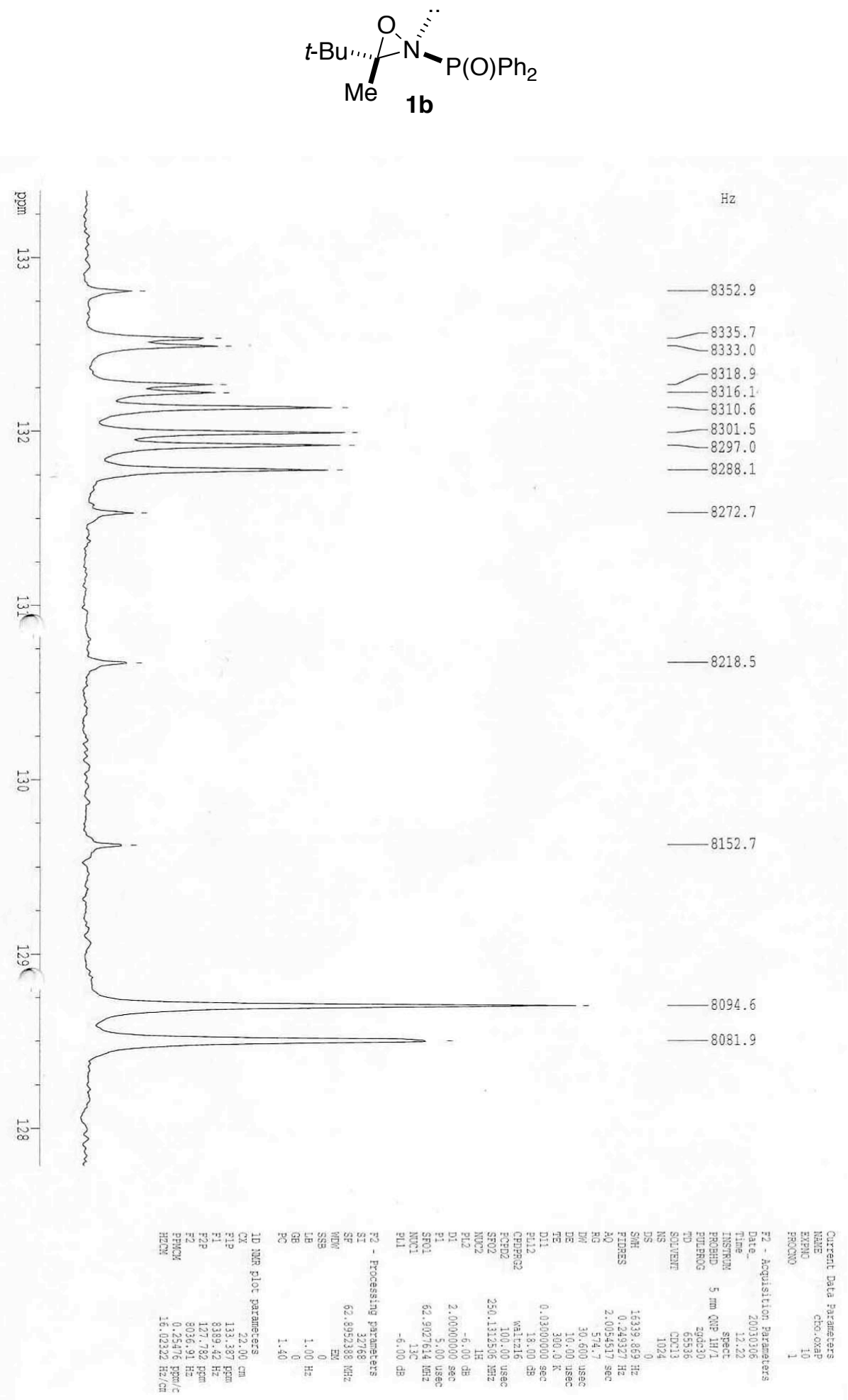
S23

${ }^{31} \mathrm{P}$ NMR $\left(\mathrm{CDCl}_{3}, 162 \mathrm{MHz}\right)$ of $1 \mathrm{~b}$
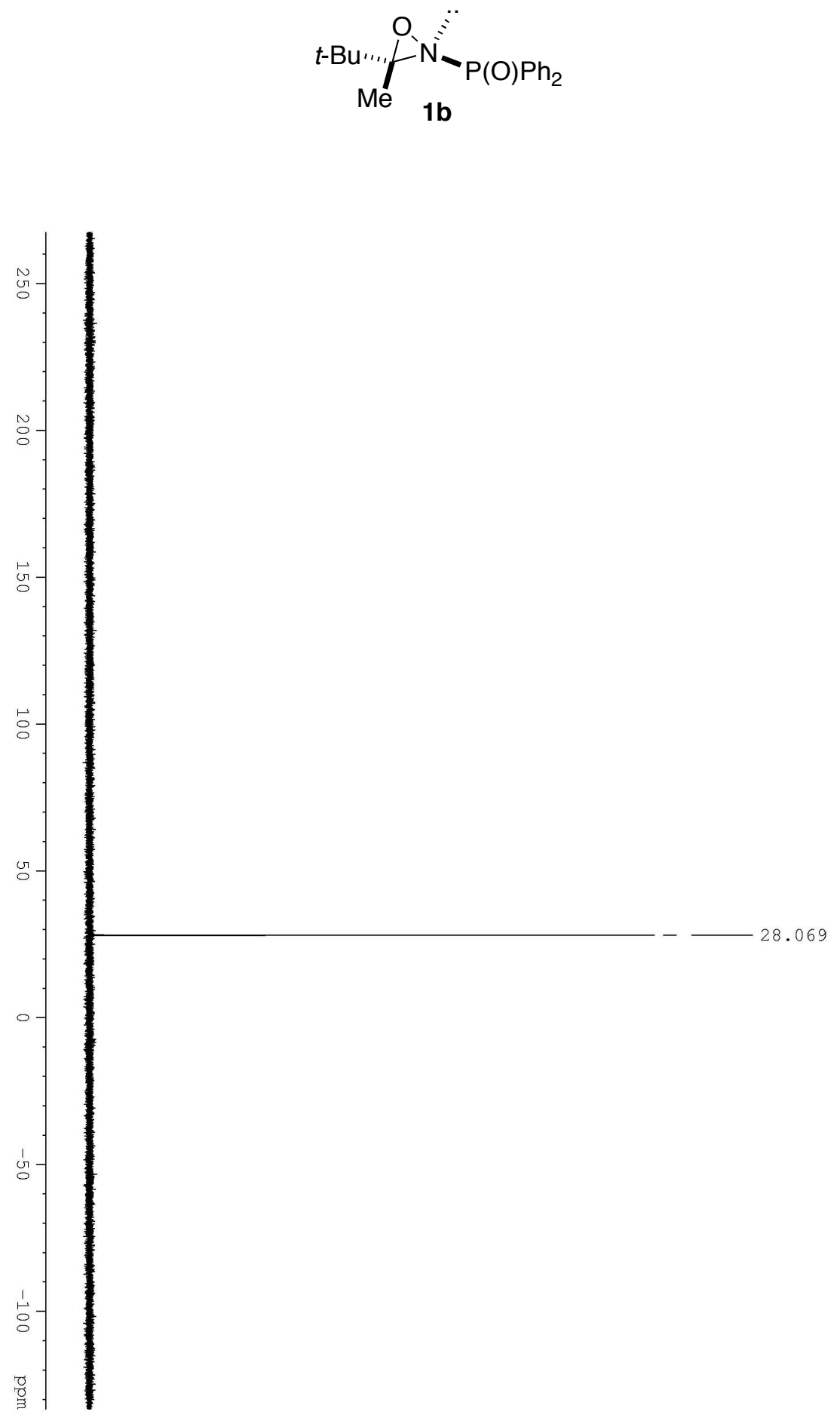


\section{${ }^{1} \mathrm{H}$ NMR $\left(\mathrm{CDCl}_{3}, 250 \mathrm{MHz}\right)$ of crude $4 \mathrm{c}$}
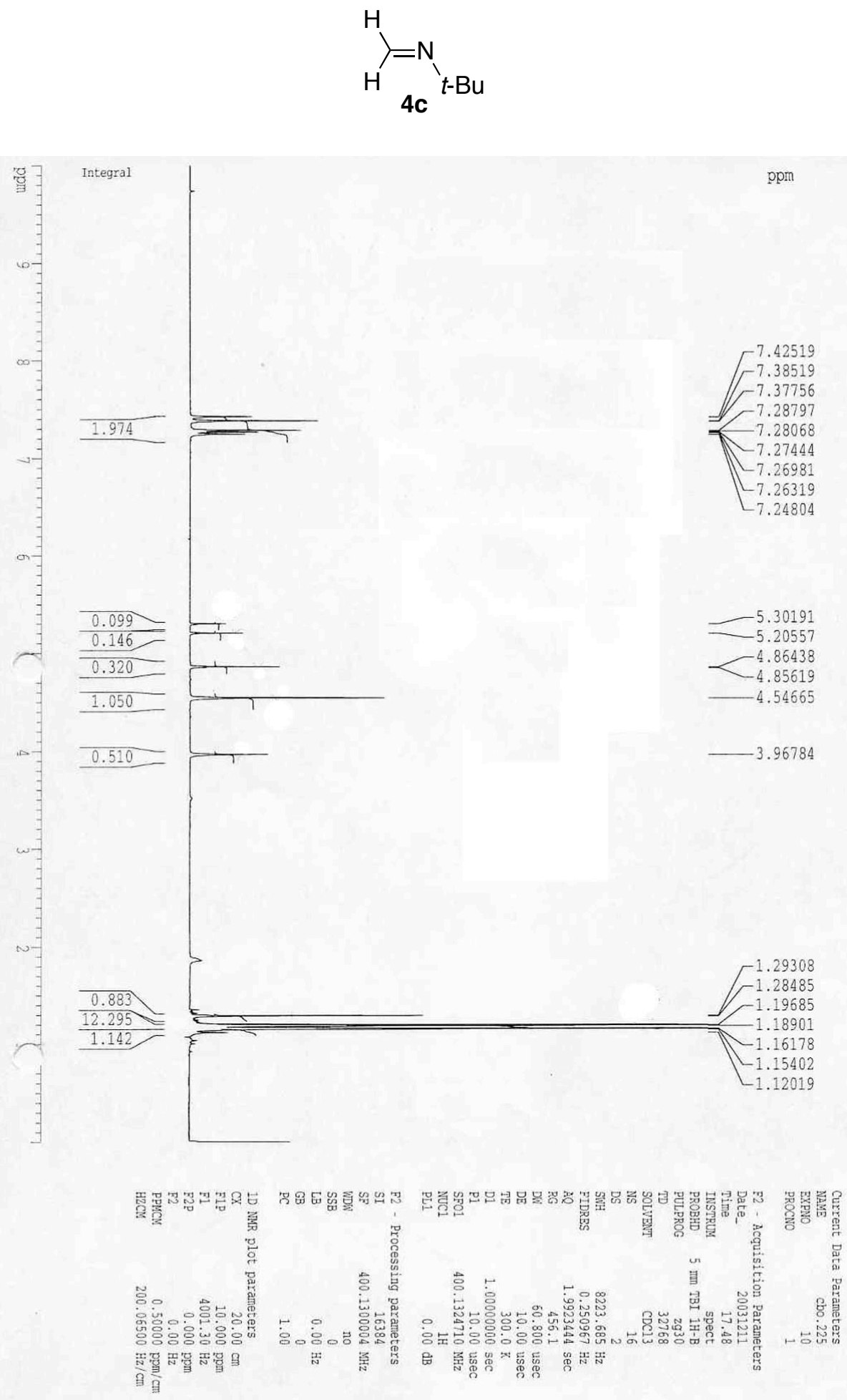
S25

${ }^{13} \mathrm{C}$ NMR $\left(\mathrm{CDCl}_{3}, 63 \mathrm{MHz}\right)$ of $4 \mathrm{c}$<smiles>C=NC(C)(C)C</smiles>
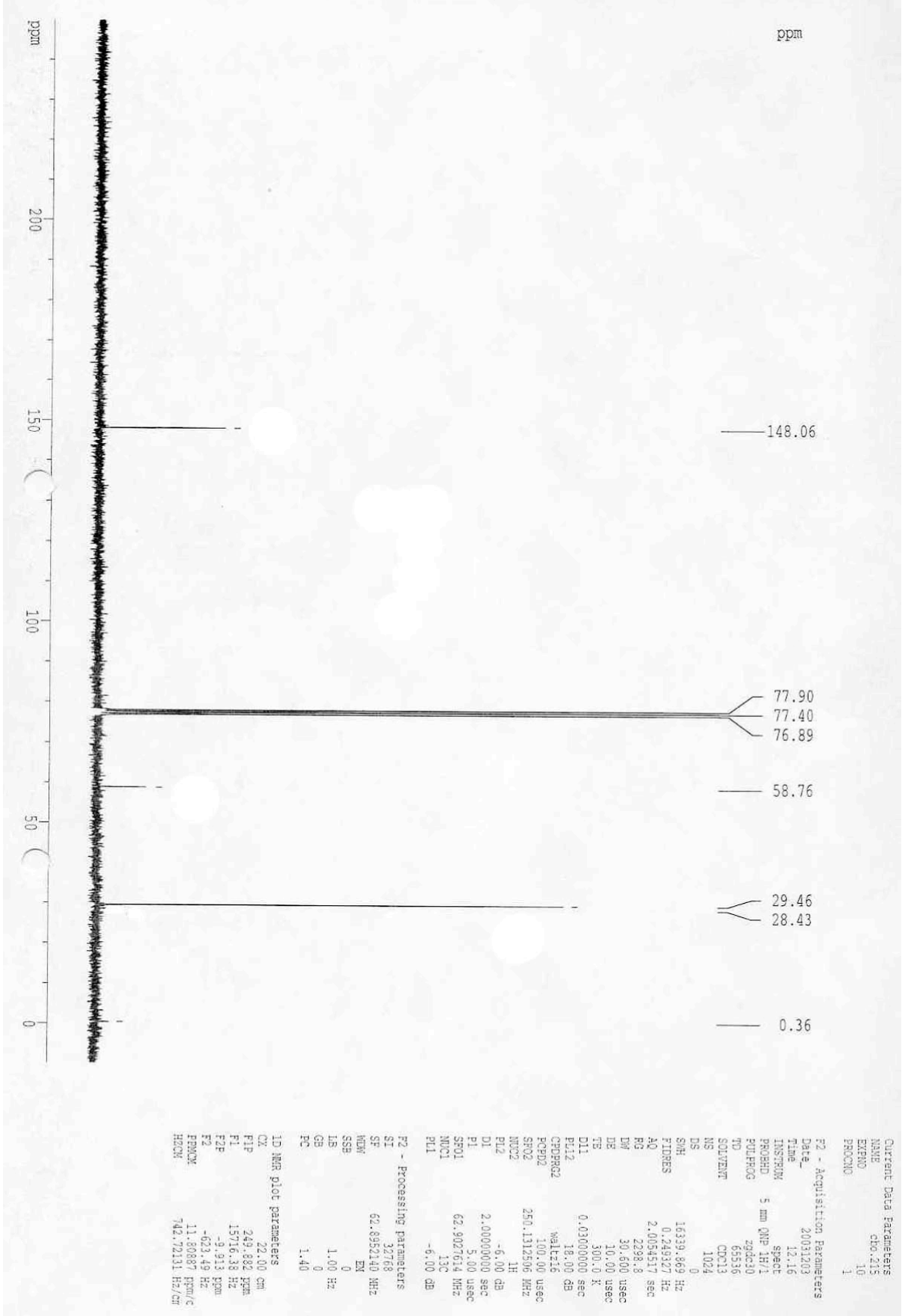


\section{${ }^{1} \mathrm{H}$ NMR $\left(\mathrm{CDCl}_{3}, 250 \mathrm{MHz}\right)$ of $1 \mathrm{c}$}
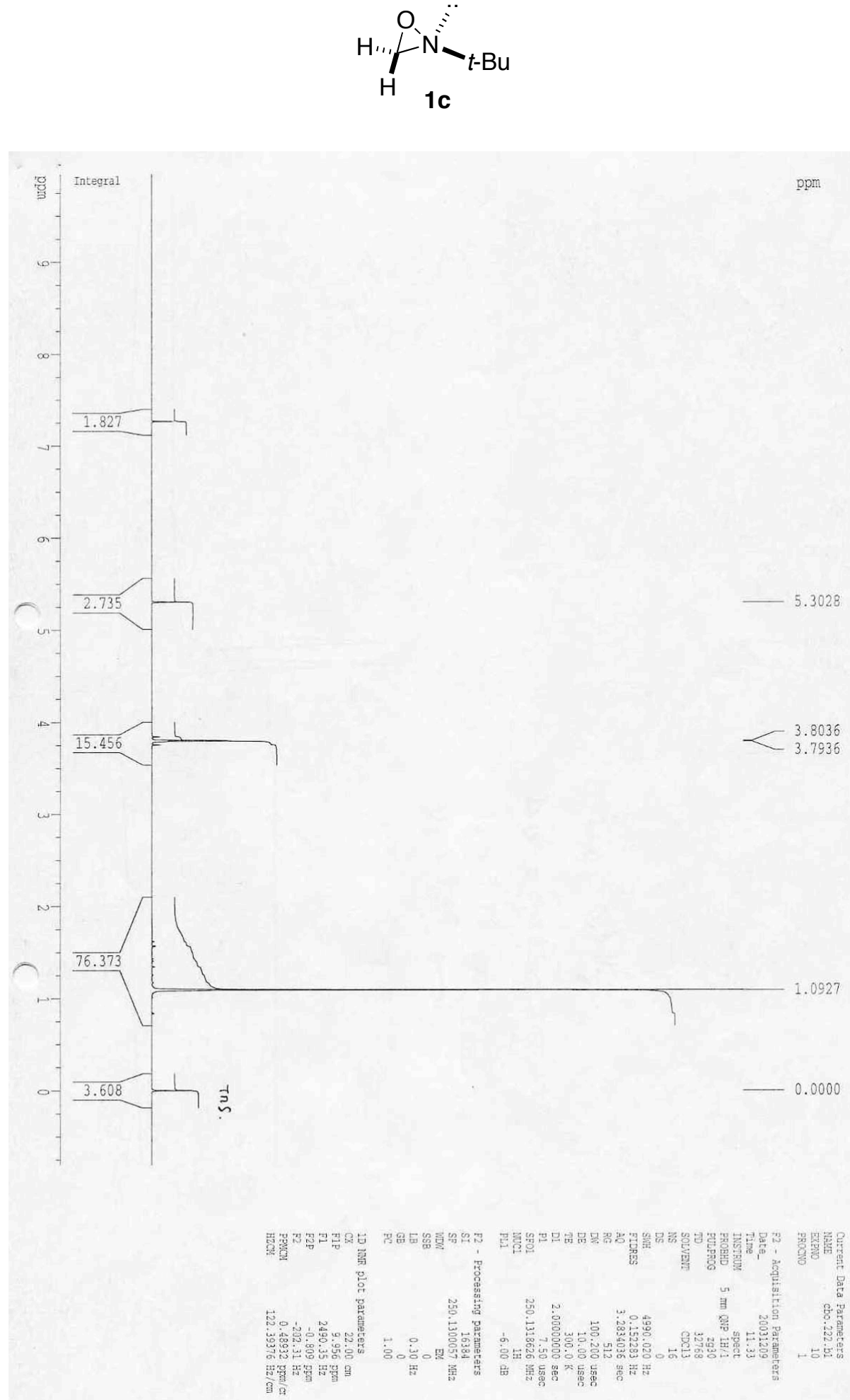


\section{${ }^{13} \mathrm{C} \mathrm{NMR}\left(\mathrm{CDCl}_{3}, 100 \mathrm{MHz}\right)$ of $1 \mathrm{c}$}

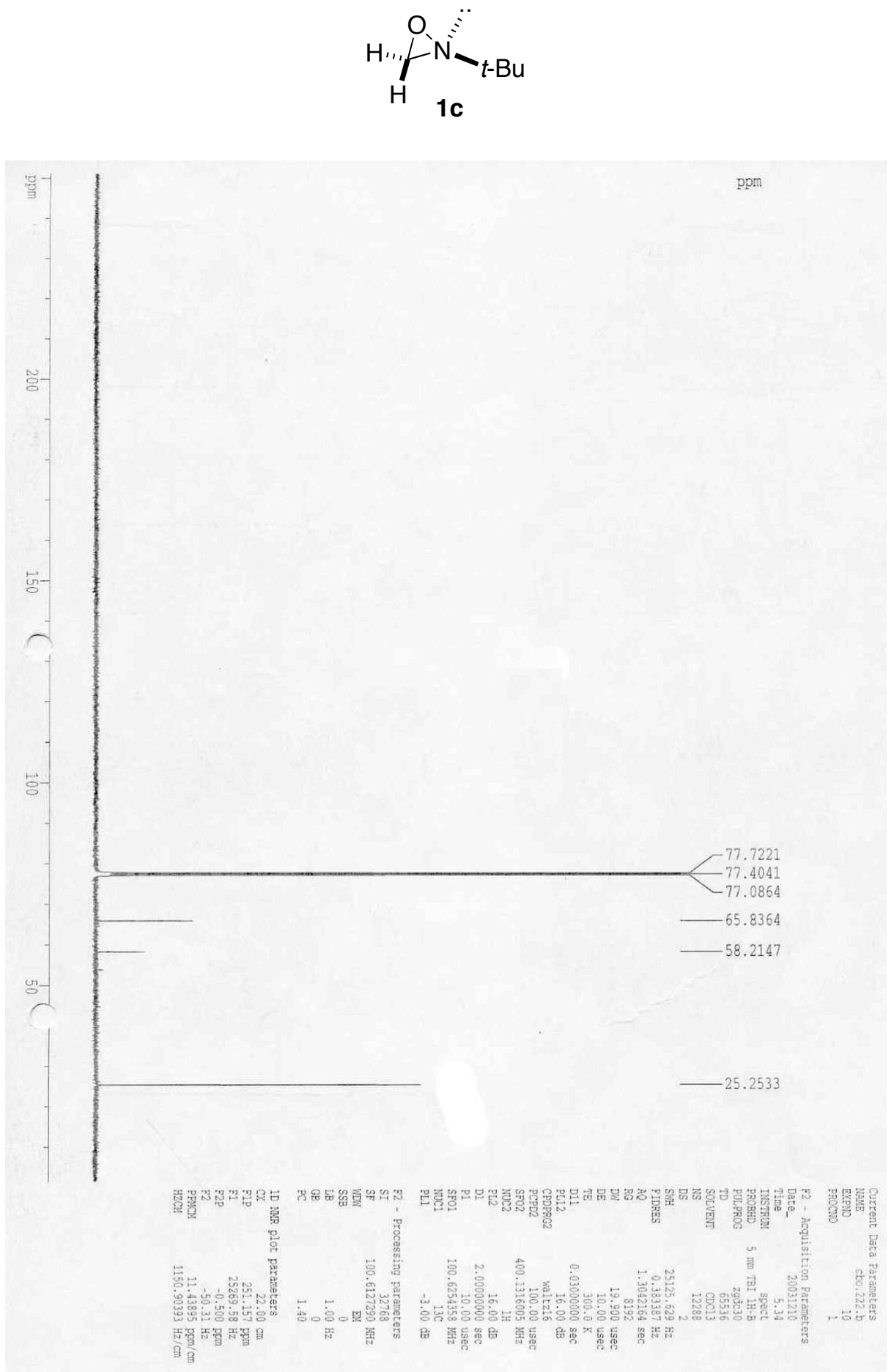


${ }^{1} \mathrm{H}$ NMR $\left(\mathrm{CDCl}_{3}, 250 \mathrm{MHz}\right)$ of $4 \mathrm{~d}$
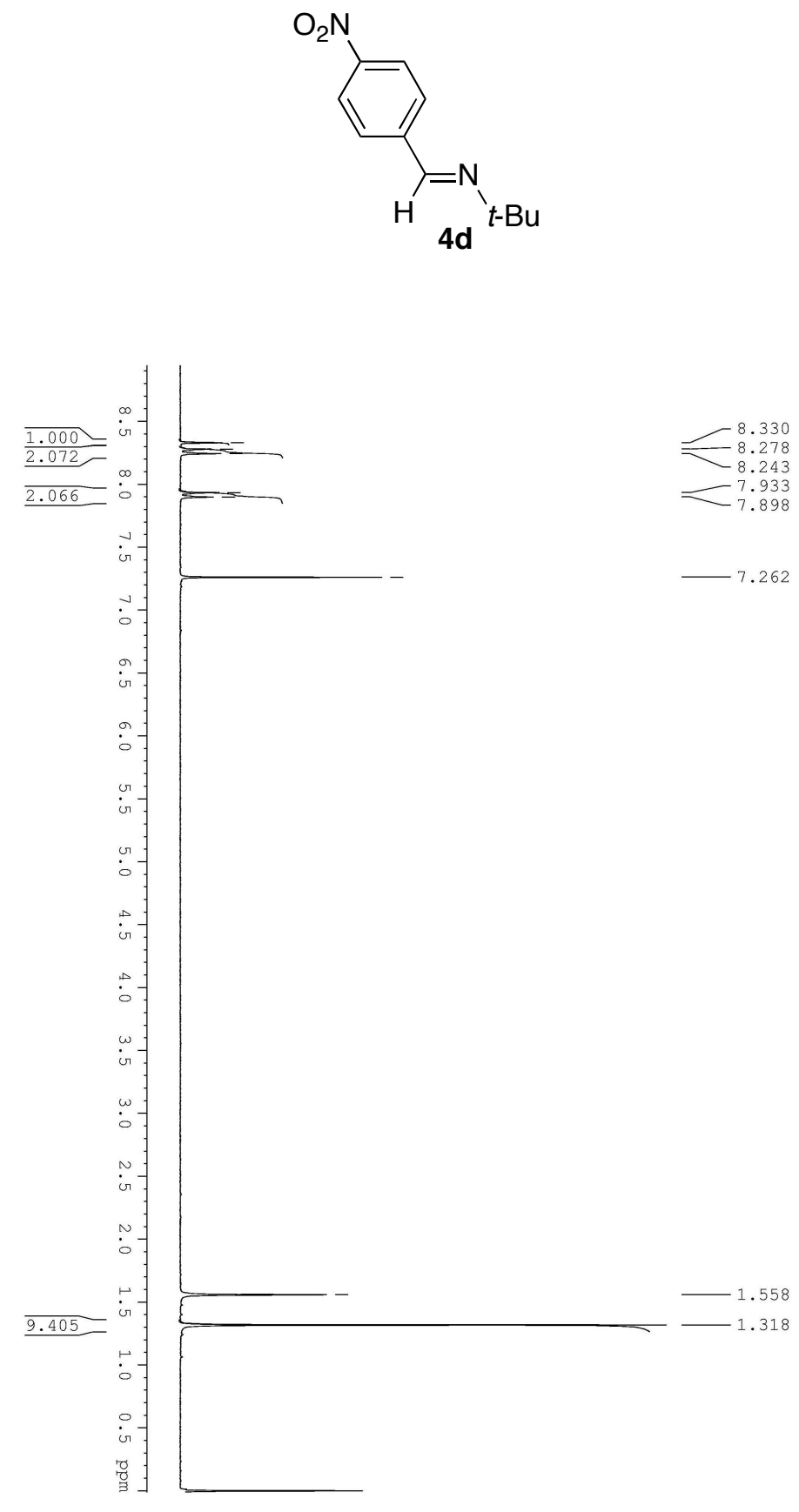
${ }^{13} \mathrm{C} \mathrm{NMR}\left(\mathrm{CDCl}_{3}, 63 \mathrm{MHz}\right)$ of $4 \mathrm{~d}$
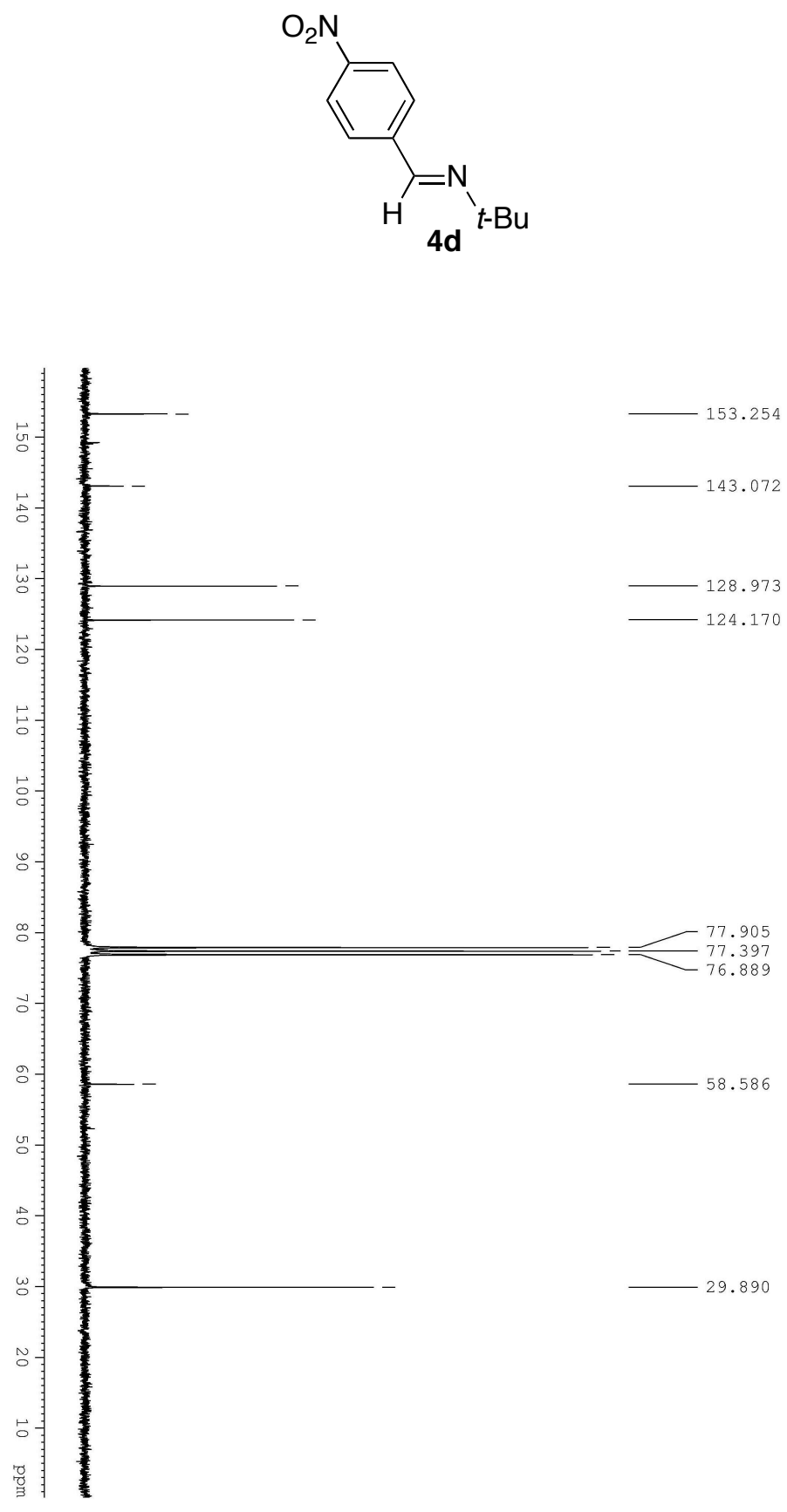
${ }^{1} \mathrm{H}$ NMR $\left(\mathrm{CDCl}_{3}, 250 \mathrm{MHz}\right)$ of $1 \mathrm{~d}$

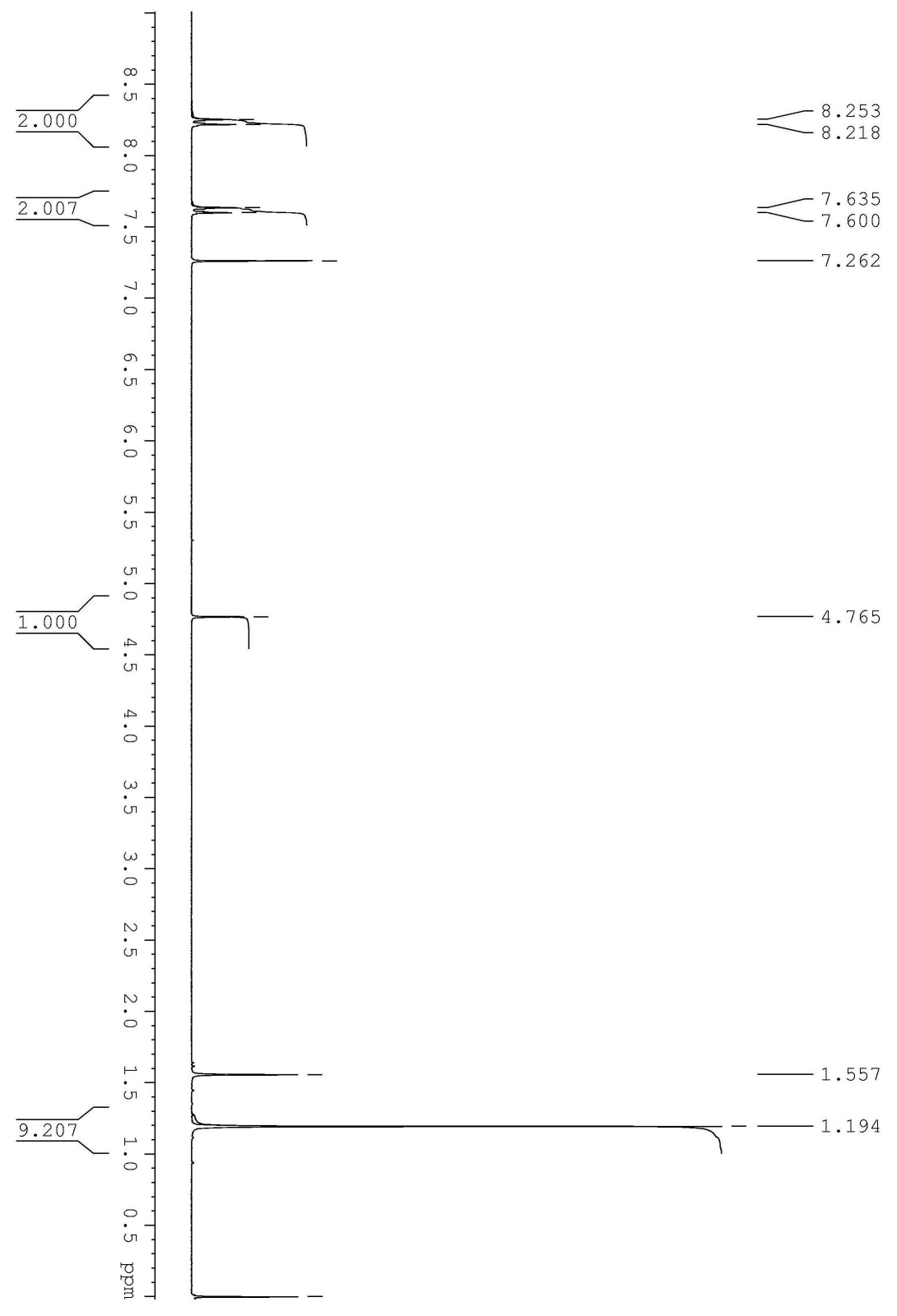


${ }^{13} \mathrm{C}$ NMR $\left(\mathrm{CDCl}_{3}, 63 \mathrm{MHz}\right)$ of $1 \mathrm{~d}$
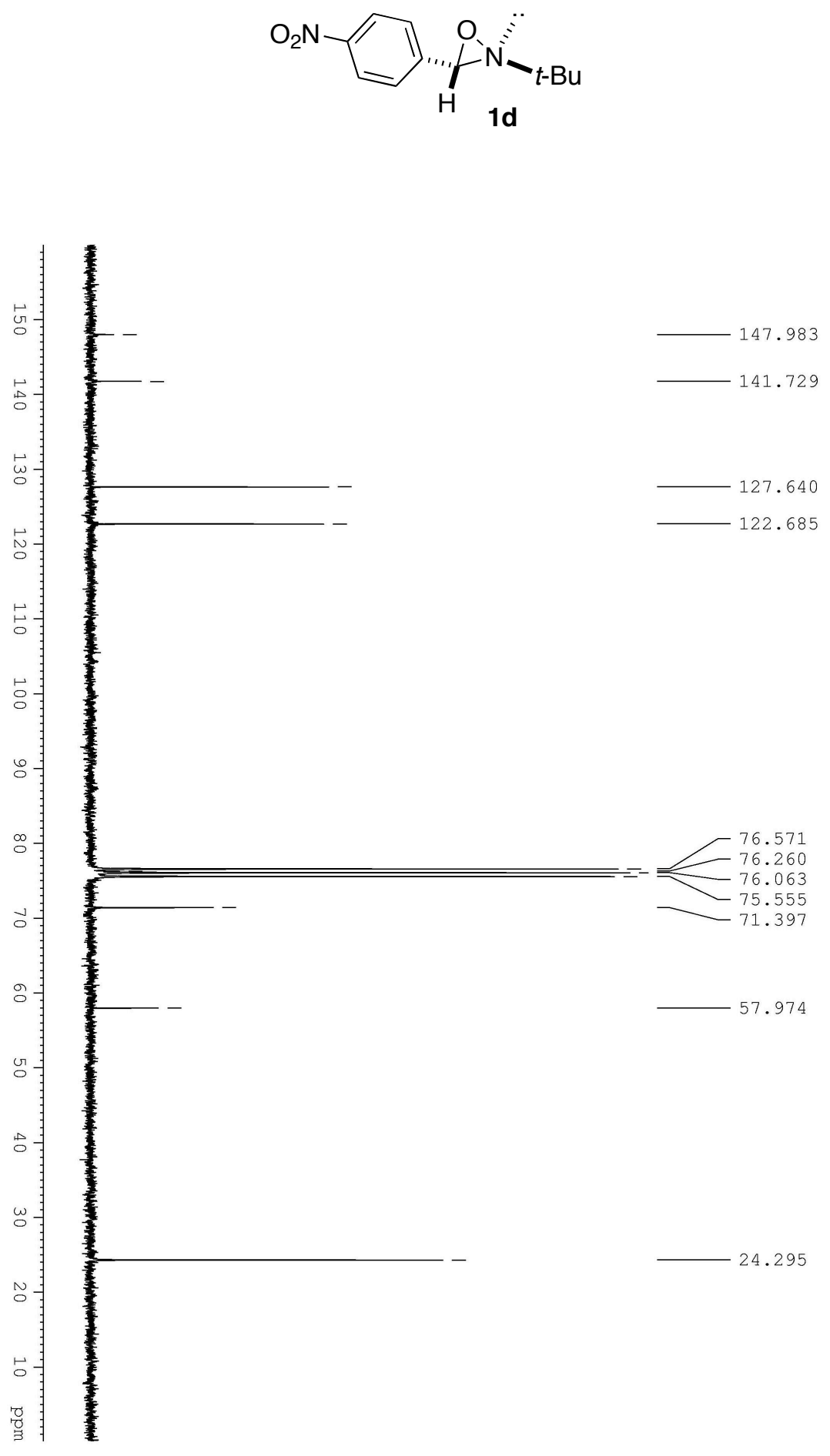


\section{${ }^{1} \mathrm{H}$ NMR $\left(\mathrm{CDCl}_{3}, 250 \mathrm{MHz}\right)$ of $4 \mathrm{e}$}
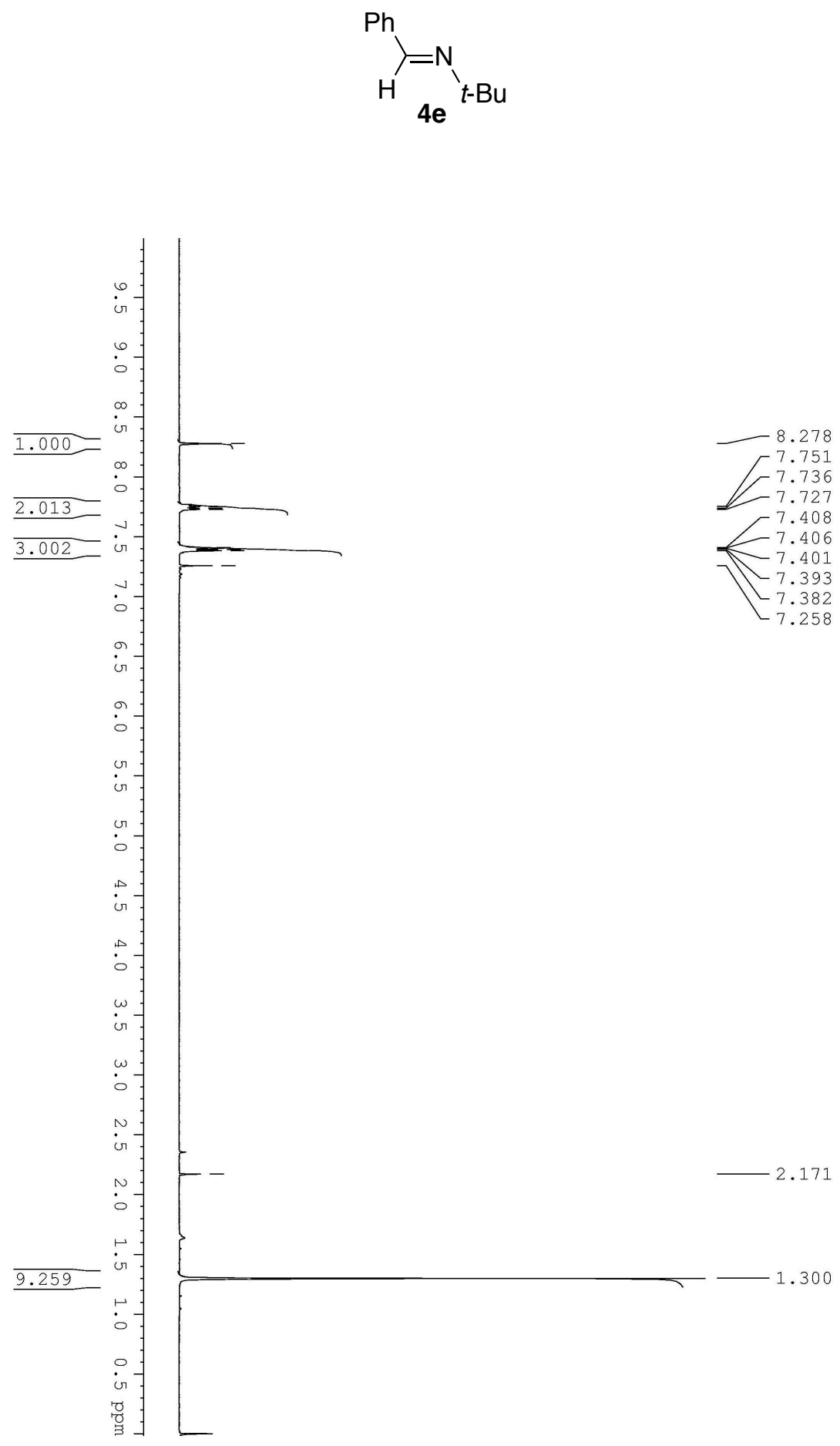
${ }^{13} \mathrm{C}$ NMR $\left(\mathrm{CDCl}_{3}, 63 \mathrm{MHz}\right)$ of $4 \mathrm{e}$
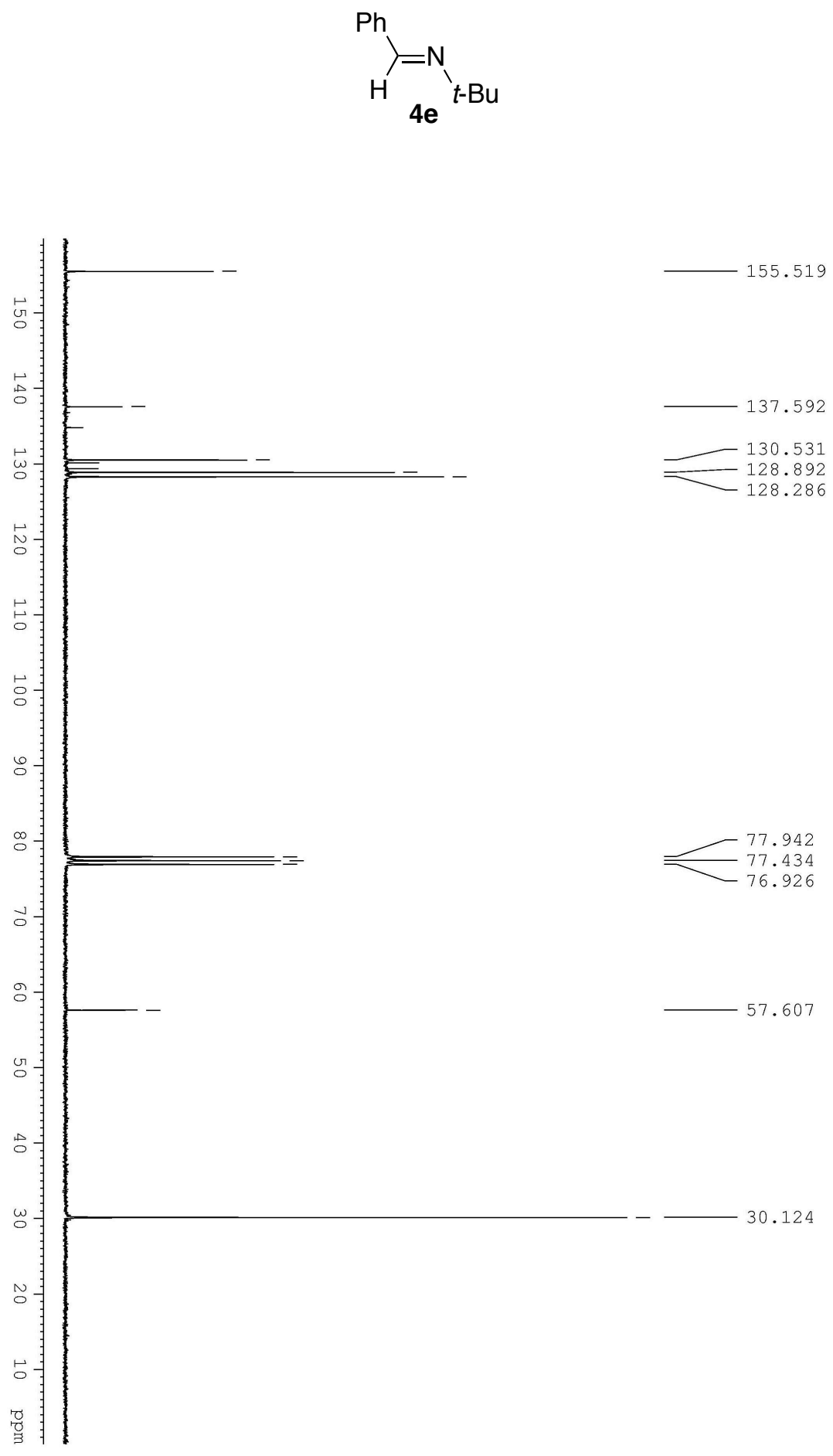


\section{${ }^{1} \mathrm{H}$ NMR $\left(\mathrm{CDCl}_{3}, 250 \mathrm{MHz}\right)$ of $1 \mathrm{e}$}

$$
{ }_{\mathrm{H}}^{\mathrm{O}} \stackrel{\mathrm{O}}{\mathrm{Ph}}
$$

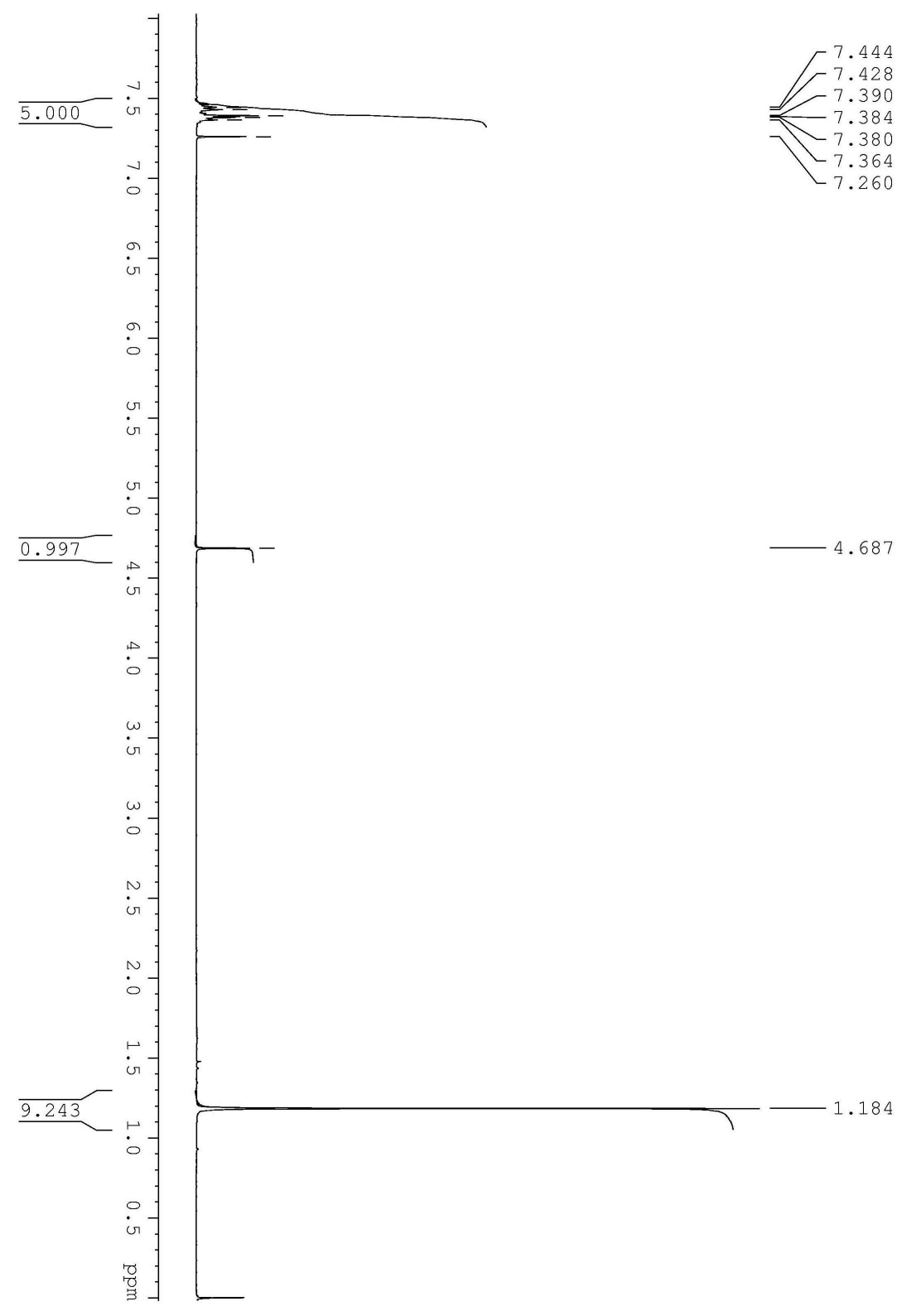


${ }^{13} \mathrm{C} \mathrm{NMR}\left(\mathrm{CDCl}_{3}, 63 \mathrm{MHz}\right)$ of $1 \mathrm{e}$
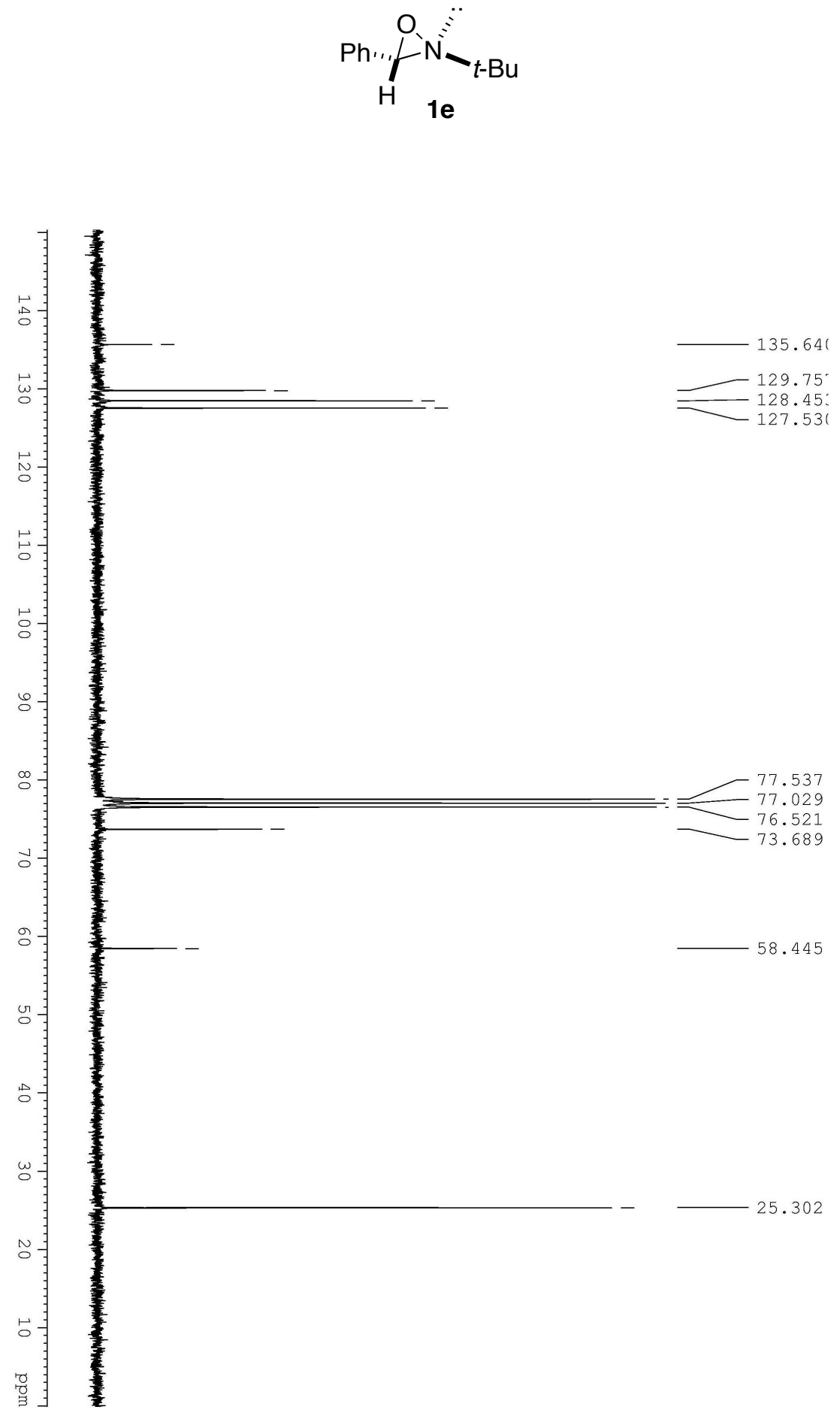